\title{
Gold(I)-Catalyzed Dynamic Kinetic Enantioselective Hydroamination of Allenes
}

\author{
Zhibin Zhang, Christopher F. Bender, and Ross A. Widenhoefer*
}

\author{
Duke University \\ French Family Science Center \\ Durham, NC 27708-0346 \\ rwidenho@chem.duke.edu
}

Experimental procedures, spectroscopic and analytical data for new compounds, crystallographic and collection data for $(R, S)$-S13 (33 pages). 


\section{Experimental}

General Methods. Reactions were performed under a nitrogen atmosphere employing standard Schlenk and drybox techniques unless specified otherwise. NMR spectra were obtained on Varian spectrometers operating at 400 or $500 \mathrm{MHz}$ for ${ }^{1} \mathrm{H} \mathrm{NMR}$ and 101 or $126 \mathrm{MHz}$ for ${ }^{13} \mathrm{C}$ NMR in $\mathrm{CDCl}_{3}$ at $25^{\circ} \mathrm{C}$ or $55^{\circ} \mathrm{C}$ and are noted accordingly. IR spectra were obtained on a Nicolet Avatar 360FT IR spectrometer. Gas chromatography was performed on a Hewlett-Pakard 5890 gas chromatography equipped with a $15 \mathrm{~m}$ or $25 \mathrm{~m}$ polydimethylsiloxane capillary column and FID detector. HPLC was performed on a Hewlett-Packard 1090Il chromatograph equipped with a $25 \mathrm{~cm}$ Chiralpak AD-H column. Column chromatography was performed employing 230-400 mesh silica gel (Silicycle). Catalytic reactions were performed in sealed glass tubes under an atmosphere of dry nitrogen unless noted otherwise. Elemental analyses were performed by Complete Analysis Laboratories (Parsippany, NJ). Thin layer chromatography (TLC) was performed on silica gel $60 \mathrm{~F}_{254}$ (EMD Chemicals Inc.). Room temperature is $23^{\circ} \mathrm{C}$.

All solvents were purchased from Aldrich or Acros in anhydrous form and used as received. All reagents were purchased from major suppliers and used as received. (S)-3-Methyl-1-pentyn-3-ol (44\% ee) was obtained by recrystallization of the corresponding phthalate half-ester with brucine employing a literature procedure. ${ }^{1}$ Racemic benzyl 4,4-diphenyl-2-vinylpyrrolidine-1-carboxylate (S12), ${ }^{2}(S)-1,{ }^{3}$ and $\mathrm{Au}\left[\mathrm{P}(t-\mathrm{Bu})_{2} \mathrm{O}\right.$-biphenyl]Cl ${ }^{4}$ were prepared by previously reported methods. Samples of racemic pyrrolidines were prepared via cyclization of the corresponding chiral $N$-allenyl carbamates catalyzed by a 1:1 mixture of $\mathrm{Au}\left[\mathrm{P}(t-\mathrm{Bu})_{2} \mathrm{O}\right.$-biphenyl] $\mathrm{Cl}$ and $\mathrm{AgOTf}$ employing a published procedure. ${ }^{5}$

\section{Chiral $\mathbf{N}$-Allenyl Carbamates}

Benzyl (6-methyl-2,2-diphenyl-4,5-octadienyl)carbamate (2a). $\quad \mathrm{TsOH} \cdot \mathrm{H}_{2} \mathrm{O}(10 \mathrm{mg}, 0.053$ mmol) was added to a solution of 3-methyl-1-pentyn-3-ol $(4.90 \mathrm{~g}, 49.9 \mathrm{mmol})$ and 3,4-dihydro-2H-pyran (5.84 g, $69.4 \mathrm{mmol})$ in $\mathrm{CH}_{2} \mathrm{Cl}_{2}(40 \mathrm{~mL})$ at $-20{ }^{\circ} \mathrm{C}$, the resulting solution was stirred for $2.5 \mathrm{~h}$ at $-20{ }^{\circ} \mathrm{C}$ and overnight at $0{ }^{\circ} \mathrm{C}$. The resulting mixture was diluted with $\mathrm{Et}_{2} \mathrm{O}(10 \mathrm{~mL})$, quenched with saturated 
$\mathrm{NaHCO}_{3}(40 \mathrm{~mL})$, and extracted with ether $(50 \mathrm{~mL})$ and $\mathrm{CH}_{2} \mathrm{Cl}_{2}(50 \mathrm{~mL})$. The combined organic extracts were dried $\left(\mathrm{MgSO}_{4}\right)$ and concentrated and the resulting oily residue was distilled under reduced pressure $\left(52{ }^{\circ} \mathrm{C}, 5\right.$ Torr) to give 2-(3-methylpent-1-yn-3-yloxy)-tetrahydro-2H-pyran (S1) as a colorless oil $(6.83 \mathrm{~g}, 75 \%)$.

A solution of $n$-BuLi $(2.5 \mathrm{M}$ in hexanes, $19.0 \mathrm{~mL}, 47.5 \mathrm{mmol})$ was added over $30 \mathrm{~min}$ to a solution of $\mathbf{S} 1(6.40 \mathrm{~g}, 35.1 \mathrm{mmol})$ in THF $(100 \mathrm{~mL})$ at $-78^{\circ} \mathrm{C}$. The resulting mixture was warmed to 0 ${ }^{\circ} \mathrm{C}$ and HMPA $(3.7 \mathrm{~mL})$ was added followed by paraformaldehyde $(2.12 \mathrm{~g}, 70.7 \mathrm{mmol})$. The reaction mixture was warmed slowly to room temperature and stirred overnight. The resulting mixture was cooled to $0{ }^{\circ} \mathrm{C}$, quenched with saturated aqueous $\mathrm{NH}_{4} \mathrm{Cl}$, and extracted with ether $(2 \times 25 \mathrm{~mL})$. The organic extracts were dried $\left(\mathrm{MgSO}_{4}\right)$ and concentrated. The resulting residue was chromatographed (EtOAc-hexanes = 1:2) to yield 4-methyl-4-(tetrahydro-2H-pyran-2-yloxy)hex-2-yn-1-ol (S2) as a colorless oil $(5.51 \mathrm{~g}, 74 \%)$.

A solution of $\mathbf{S 2}(5.50 \mathrm{~g}, 25.9 \mathrm{mmol})$ in ether $(10 \mathrm{~mL})$ was added to a stirred suspension of $\mathrm{LiAlH}_{4}(2.25 \mathrm{~g}, 59.3 \mathrm{mmol})$ in ether $(110 \mathrm{~mL})$ at $0{ }^{\circ} \mathrm{C}$ and warmed to room temperature overnight. The reaction mixture was cooled to $0{ }^{\circ} \mathrm{C}$ and quenched by successive addition of water $(3.15 \mathrm{~mL}), 15 \%$ $\mathrm{NaOH}[(\mathrm{w} / \mathrm{w}), 3.15 \mathrm{~mL}]$, and water $(3.15 \mathrm{~mL})$. The resulting white suspension was filtered through Celite, eluted with ether, and concentrated. The resulting residue was chromatographed $\left(\mathrm{Et}_{2} \mathrm{O}-\mathrm{CH}_{2} \mathrm{Cl}_{2}\right.$ $=1: 19)$ to yield 4-methyl-2,3-hexadien-1-ol (S3) as a colorless oil ( $2.20 \mathrm{~g}, 76 \%)$.

Methanesulfonyl chloride $(1.45 \mathrm{~mL}, 18.7 \mathrm{mmol})$ was added dropwise to a solution of $\mathbf{S 3}(1.68 \mathrm{~g}$, $15.0 \mathrm{mmol})$, DMAP (185 mg, $1.51 \mathrm{mmol})$, and $\mathrm{Et}_{3} \mathrm{~N}(3.20 \mathrm{~mL}, 22.8 \mathrm{mmol})$ in $\mathrm{CH}_{2} \mathrm{Cl}_{2}(50 \mathrm{~mL})$ at $0{ }^{\circ} \mathrm{C}$. The resulting suspension was stirred at $0{ }^{\circ} \mathrm{C}$ for $1 \mathrm{~h}$, treated with water $(40 \mathrm{~mL})$, and extracted with $\mathrm{CH}_{2} \mathrm{Cl}_{2}(3 \times 25 \mathrm{~mL})$. The combined organic extracts were washed with $1 \mathrm{M} \mathrm{HCl}(25 \mathrm{~mL})$, saturated aqueous sodium bicarbonate $(25 \mathrm{~mL})$, and brine $(25 \mathrm{~mL})$, dried $\left(\mathrm{MgSO}_{4}\right)$, and concentrated to give 4methyl-2,3-hexadienyl methanesulfonate (S4) as a yellow oil that was used in the subsequent step without further purification. 
A solution of diphenylacetonitrile $(2.33 \mathrm{~g}, 12.1 \mathrm{mmol})$ in DMF $(20 \mathrm{~mL})$ was added dropwise to a suspension of $\mathrm{NaH}(95 \%, 427 \mathrm{mg}, 16.9 \mathrm{mmol})$ in THF $(40 \mathrm{~mL})$ at $0{ }^{\circ} \mathrm{C}$. The resulting mixture was stirred vigorously for $1 \mathrm{~h}$, treated sequentially with a solution of crude $\mathbf{S 4}$ (all material obtained in previous step) in DMF (15 mL) and a single portion of Nal $(2.15 \mathrm{~g}, 14.3 \mathrm{mmol})$. The resulting solution was warmed to room temperature overnight with stirring. The resulting mixture was treated with water $(60 \mathrm{~mL})$ and extracted with ether $(3 \times 40 \mathrm{~mL})$. The combined organic extracts were washed with water $(50 \mathrm{~mL})$ and brine $(25 \mathrm{~mL})$, dried $\left(\mathrm{MgSO}_{4}\right)$, concentrated, and the resulting residue was chromatographed (EtOAc-hexanes $=1: 25)$ to give 6-methyl-2,2-diphenyl-4,5-octadienenitrile (S5) as a colorless, viscous oil $(2.48 \mathrm{~g}, 71 \%)$.

A solution of DIBAL-H (1 M in toluene, $7.50 \mathrm{~mL}, 7.50 \mathrm{mmol})$ was added to a solution of $\mathbf{S 5}(1.47$ $\mathrm{g}, 5.11 \mathrm{mmol})$ in ether $(35 \mathrm{~mL})$ at $-42{ }^{\circ} \mathrm{C}$ over $10 \mathrm{~min}$ and stirred for $3 \mathrm{~h}$. $\mathrm{NaBH}_{4}(572 \mathrm{mg}, 15.1 \mathrm{mmol})$ was added in one portion to the resulting solution followed by the slow addition of EtOH (30 $\mathrm{mL})$ over $15 \mathrm{~min}$. The reaction mixture was warmed to $0^{\circ} \mathrm{C}$ and stirred vigorously for $3 \mathrm{~h}$, treated with saturated aqueous potassium carbonate $(20 \mathrm{~mL})$, and extracted with ether $(50 \mathrm{~mL})$. The organic extracts were washed with saturated potassium carbonate $(20 \mathrm{~mL})$ and brine $(20 \mathrm{~mL})$, dried $\left(\mathrm{MgSO}_{4}\right)$, and concentrated to give crude 6-methyl-2,2-diphenyl-4,5-octadien-1-amine (S6) as a viscous oil that was used in the subsequent step without further purification.

Benzyl chloroformate $(1.35 \mathrm{~mL}, 6.15 \mathrm{mmol}$ ) was added to a suspension of crude $\mathbf{S 6}$ (all material obtained in previous step) and $\mathrm{NaHCO}_{3}(630 \mathrm{mg}, 7.50 \mathrm{mmol})$ in $\mathrm{EtOH} /$ water $[2 / 1(\mathrm{v} / \mathrm{v}), 54 \mathrm{~mL}]$ at $0{ }^{\circ} \mathrm{C}$. The resulting mixture was warmed slowly to room temperature and stirred vigorously overnight. The reaction mixture was diluted with ether $(100 \mathrm{~mL})$, washed with water $(2 \times 30 \mathrm{~mL})$ and brine $(30 \mathrm{~mL})$, dried $\left(\mathrm{MgSO}_{4}\right)$, and concentrated. The resulting residue was chromatographed (EtOAc-hexanes = $1: 12)$ to give $2 \mathrm{a}(1.33 \mathrm{~g}, 61 \%$ from $\mathbf{S 5})$ as colorless, viscous oil. 
For S3. ${ }^{6}$ TLC (ether- $\left.\mathrm{CH}_{2} \mathrm{Cl}_{2}=1: 19\right): \quad R_{f}=0.39 .{ }^{1} \mathrm{H}$ NMR: $\delta \quad 5.22-5.28(\mathrm{~m}, 1 \mathrm{H}), 4.03(\mathrm{~d}, \mathrm{~J}=$ $5.6 \mathrm{~Hz}, 2 \mathrm{H}), 1.93(\mathrm{dq}, J=3.2,7.3 \mathrm{~Hz}, 2 \mathrm{H}), 1.68(\mathrm{~d}, J=2.9 \mathrm{~Hz}, 3 \mathrm{H}), 1.68(\mathrm{br} \mathrm{s}, 1 \mathrm{H}), 0.96(\mathrm{t}, J=7.3$ $\mathrm{Hz}, 3 \mathrm{H})$.

For S5. TLC (EtOAc-hexanes = 1:25): $R_{f}=0.24 .{ }^{1} \mathrm{H}$ NMR: $\delta \quad 7.27-7.43(\mathrm{~m}, 10 \mathrm{H})$, 4.98-5.06 $(\mathrm{m}, 1 \mathrm{H}), 3.08(\mathrm{~d}, J=7.0 \mathrm{~Hz}, 2 \mathrm{H}), 1.77-1.93(\mathrm{~m}, 2 \mathrm{H}), 1.55(\mathrm{~d}, J=2.7 \mathrm{~Hz}, 3 \mathrm{H}), 0.90(\mathrm{t}, J=7.5 \mathrm{~Hz}, 3$ H). ${ }^{13} \mathrm{C}\left\{{ }^{1} \mathrm{H}\right\}$ NMR: $\delta 203.5,140.0,128.9,127.9,127.3,127.2,122.3,84.9,76.8,52.1,40.7,26.9,18.6$, 12.2. IR (neat, $\mathrm{cm}^{-1}$ ): 2966, 1964, 1493, 1447, 753, 695. Anal. calcd (found) for $\mathrm{C}_{21} \mathrm{H}_{21} \mathrm{~N}: \mathrm{H}, 7.36$ (7.36); C, 87.76 (87.67).

For 2a: TLC (EtOAc-hexanes = 1:12): $R_{f}=0.21 .{ }^{1} \mathrm{H}$ NMR $\left(55^{\circ} \mathrm{C}\right): \quad \delta \quad 7.14-7.33(\mathrm{~m}, 15 \mathrm{H})$, 5.01 (s, 2 H), 4.70 (br s, 1 H), 4.38 (br s, 1 H), 3.98 (d, J = 5.8 Hz, 2 H), 2.80 (d, J = 7.5 Hz, 2 H), 1.73$1.88(\mathrm{~m}, 2 \mathrm{H}), 1.50(\mathrm{~d}, J=2.9 \mathrm{~Hz}, 3 \mathrm{H}), 0.87(\mathrm{t}, J=7.5 \mathrm{~Hz}, 3 \mathrm{H}) .{ }^{13} \mathrm{C}\left\{{ }^{1} \mathrm{H}\right\} \operatorname{NMR}\left(55^{\circ} \mathrm{C}\right): \delta 203.0,156.5$, $145.4,145.3,136.9,128.6,128.4,128.4,128.3,128.3,128.2,128.2,126.6,100.9,85.9,66.8,50.8$ $48.5,38.7,27.1,18.6,12.4$. IR (neat, $\mathrm{cm}^{-1}$ ): $2963,1718,1505,1447,1213,696$. Anal. calcd (found) for $\mathrm{C}_{29} \mathrm{H}_{31} \mathrm{NO}_{2}: \mathrm{H}, 7.52$ (7.27); C, 81.85 (81.59).

(R)-2a (44\% ee). (S)-(+)-3-Methyl-1-pentyn-3-ol ( 50\% ee) was obtained by recrystallization of the corresponding phthalate half-ester with brucine employing a published procedure. ${ }^{1}$ The absolute configuration of $(S)-(+)-3-m e t h y l-1-p e n t y n-3-o l$ was established by Faulkner. ${ }^{7} \quad(S)-(+)-3-M e t h y l-1-$ pentyn-3-ol ( $\sim 50 \%$ ee) was converted to $(R)-2$ a $\left(44 \%\right.$ ee, Figure S1) via $\mathrm{LiAlH}_{4}$ reduction of $(S)$-S2 employing a synthesis analogous to that used to prepare rac-2a from ( \pm )-3-methyl-1-pentyn-3-ol. The configuration of the allene is set by delivery of the hydride to the same face as the THP leaving group via trans addition of the $\mathrm{Al}-\mathrm{H}$ bond across the $\mathrm{C} \equiv \mathrm{C}$ bond followed by anti elimination (Scheme S1). ${ }^{8}$

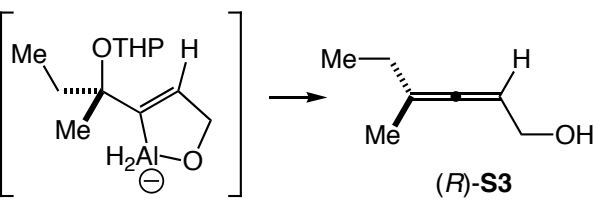

\section{Scheme S1}


Benzyl (6-methyl-2,2-diphenyl-4,5-dodecadienyl)carbamate $\quad(\mathbf{2 b}), \quad$ benzyl $\quad$ (6,8-dimethyl-2,2diphenyl-4,5-nonadienyl)carbamate (2c), benzyl (6,7-dimethyl-2,2-diphenyl-4,5-octadienyl)carbamate (2d), and benzyl (6,7,7-trimethyl-2,2-diphenyl-4,5-octadienyl)-carbamate (2e) were synthesized employing a protocol similar to that used to synthesize 2 a by starting with 3-methyl-1-nonyn-3-ol, 3,5dimethyl-1-hexyn-3-ol, 3,4-dimethyl-1-pentyn-3-ol, 3,4,4-trimethyl-1-pentyn-3-ol, respectively.

For 4-methyl-2,3-decadien-1-ol. TLC (ether- $\left.\mathrm{CH}_{2} \mathrm{Cl}_{2}=1: 19\right): R_{f}=0.45 .{ }^{1} \mathrm{H}$ NMR: $\delta \quad 5.18-5.24$ $(\mathrm{m}, 1 \mathrm{H}), 4.03(\mathrm{~d}, J=5.6 \mathrm{~Hz}, 2 \mathrm{H}), 1.92(\mathrm{dt}, J=2.7,7.0 \mathrm{~Hz}, 2 \mathrm{H}), 1.66(\mathrm{~d}, J=2.7 \mathrm{~Hz}, 3 \mathrm{H}), 1.50(\mathrm{~m}, 1$ $H), 1.20-1.40(m, 8 H), 0.84(t, J=6.8 \mathrm{~Hz}, 3 \mathrm{H})$.

For 6-methyl-2,2-diphenyl-4,5-dodecadienenitrile. Colorless oil, 66\%. TLC (EtOAc-hexanes = 1:25): $R_{f}=0.24 .{ }^{1} \mathrm{H}$ NMR: $\delta 7.27-7.43(\mathrm{~m}, 10 \mathrm{H}), 4.95-5.02(\mathrm{~m}, 1 \mathrm{H}), 3.07(\mathrm{~d}, J=7.0 \mathrm{~Hz}, 2 \mathrm{H}), 1.76-$ $1.89(\mathrm{~m}, 2 \mathrm{H}), 1.54(\mathrm{~d}, J=2.7 \mathrm{~Hz}, 3 \mathrm{H}), 1.22-1.33(\mathrm{~m}, 8 \mathrm{H}), 0.90(\mathrm{t}, J=7.0 \mathrm{~Hz}, 3 \mathrm{H}) .{ }^{13} \mathrm{C}\left\{{ }^{1} \mathrm{H}\right\} \mathrm{NMR}: \delta$ $204.0,140.1,140.0,128.9,128.9,127.9,127.9,127.3,127.2,122.3,100.8,84.2,52.1,40.7,33.8$, 31.8, 29.1, 27.4, 22.8, 18.7, 14.2. IR (neat, $\mathrm{cm}^{-1}$ ): 2924, 2854, 1493, 1447, 753, 695. Anal. calcd (found) for $\mathrm{C}_{25} \mathrm{H}_{29} \mathrm{~N}: \mathrm{H}, 8.51$ (8.38); C, 87.41 (87.29).

For 2b. Colorless oil, 27\% (two steps). TLC (EtOAc-hexanes $=1: 12): R_{f}=0.26 .{ }^{1} \mathrm{H}$ NMR (55 $\left.{ }^{\circ} \mathrm{C}\right): \delta$ 7.19-7.35 (m, $\left.15 \mathrm{H}\right), 5.04(\mathrm{~s}, 2 \mathrm{H}), 4.70(\mathrm{br} \mathrm{s}, 1 \mathrm{H}), 4.40(\mathrm{br} \mathrm{s}, 1 \mathrm{H}), 4.00(\mathrm{~d}, \mathrm{~J}=6.0 \mathrm{~Hz}, 2 \mathrm{H})$, $2.82(\mathrm{~d}, J=7.5 \mathrm{~Hz}, 2 \mathrm{H}), 1.74-1.86(\mathrm{~m}, 2 \mathrm{H}), 1.51(\mathrm{~d}, J=2.9 \mathrm{~Hz}, 3 \mathrm{H}), 1.24-1.34(\mathrm{~m}, 8 \mathrm{H}), 0.90(\mathrm{t}, J=$ $7.0 \mathrm{~Hz}, 3 \mathrm{H}) .{ }^{13} \mathrm{C}\left\{{ }^{1} \mathrm{H}\right\}$ NMR $\left(55^{\circ} \mathrm{C}\right): \delta 203.5,156.5,145.4,145.3,136.9,128.6,128.4,128.4,128.2$, 128.2, 126.6, 99.2, 85.2, 66.9, 50.9, 48.5, 38.7, 34.1, 31.9, 29.1, 27.7, 22.8, 18.7, 14.2. IR (neat, $\left.\mathrm{cm}^{-1}\right)$ : 2925, 1719, 1507, 1448, 1220, 696. Anal. calcd (found) for $\mathrm{C}_{33} \mathrm{H}_{39} \mathrm{NO}_{2}: \mathrm{H}, 8.16$ (8.17); C, 82.29 (82.26).

For 4,6-dimethyl-2,3-heptadien-1-ol. TLC (ether- $\mathrm{CH}_{2} \mathrm{Cl}_{2}=1: 19$ ): $\quad R_{f}=0.41 .{ }^{1} \mathrm{H} \mathrm{NMR}: \quad \delta$ 5.20-5.25 (m, $1 \mathrm{H}), 4.08(\mathrm{~d}, J=5.8 \mathrm{~Hz}, 2 \mathrm{H}), 1.84-1.87(\mathrm{~m}, 1 \mathrm{H}), 1.71-1.79(\mathrm{~m}, 1 \mathrm{H}), 1.69(\mathrm{~d}, J=2.9 \mathrm{~Hz}$, $3 \mathrm{H}), 1.40$ (br s, $1 \mathrm{H}), 0.91(\mathrm{~d}, J=6.5 \mathrm{~Hz}, 6 \mathrm{H})$. 
For 6,8-dimethyl-2,2-diphenyl-4,5-nonadienenitrile. Colorless oil, 64\%. TLC (EtOAchexanes = 1:25): $R_{f}=0.23 .{ }^{1} \mathrm{H}$ NMR: $\delta 7.27-7.43(\mathrm{~m}, 10 \mathrm{H}), 4.93-5.00(\mathrm{~m}, 1 \mathrm{H}), 3.07(\mathrm{~d}, J=7.2 \mathrm{~Hz}, 2$ $H), 1.57-1.77(m, 3 H), 1.52(d, J=3 H), 0.86(d, J=6.3 \mathrm{~Hz}, 3 \mathrm{H}), 0.85(\mathrm{~d}, J=6.3 \mathrm{~Hz}, 3 \mathrm{H}) .{ }^{13} \mathrm{C}\left\{{ }^{1} \mathrm{H}\right\}$ NMR: $\delta \quad 204.6,140.0,140.0,128.9,128.9,127.9,127.9,127.3,127.3,122.3,99.4,83.7,52.2,43.4$, 40.7, 26.3, 22.6, 22.5, 18.8. IR (neat, $\mathrm{cm}^{-1}$ ): 2953, 1966, 1493, 1448, 752, 695. HRMS calcd (found) for $\mathrm{C}_{23} \mathrm{H}_{25} \mathrm{~N}^{+}\left(\mathrm{M}^{+}\right)$: 315.1987 (315.1986). Anal. calcd (found) for $\mathrm{C}_{23} \mathrm{H}_{25} \mathrm{~N}: \quad \mathrm{H}, 7.99$ (7.85); C, 87.57 (87.46).

For 2c. Colorless oil, 22\% (two steps). TLC (EtOAc-hexanes $=1: 9): \quad R_{f}=0.21 .{ }^{1} \mathrm{H}$ NMR (55 ${ }^{\circ} \mathrm{C}$, Figure S2): $\delta$ 7.19-7.35 (m, $\left.15 \mathrm{H}\right), 5.04(\mathrm{~s}, 2 \mathrm{H}), 4.69(\mathrm{br} \mathrm{s}, 1 \mathrm{H}), 4.40(\mathrm{br} \mathrm{s}, 1 \mathrm{H}), 4.00(\mathrm{~d}, J=6.0$ Hz, $2 \mathrm{H}), 2.77-2.87(\mathrm{~m}, 2 \mathrm{H}), 1.55-1.74(\mathrm{~m}, 3 \mathrm{H}), 1.49(\mathrm{~d}, J=2.7 \mathrm{~Hz}, 3 \mathrm{H}), 0.85(\mathrm{~d}, J=6.3 \mathrm{~Hz}, 6 \mathrm{H})$. ${ }^{13} \mathrm{C}\left\{{ }^{1} \mathrm{H}\right\}$ NMR $\left(55^{\circ} \mathrm{C}\right.$, Figure S3): $\delta \quad 204.2,156.5,145.3,136.9,128.6,128.4,128.4,128.4,128.4$, 128.2, 128.2, 126.6, 126.6, 97.9, 84.7, 66.9, 50.9, 48.5, 43.7, 38.6, 26.5, 22.6, 22.6, 18.9. IR (neat, $\mathrm{cm}^{-}$ $\left.{ }^{1}\right): \quad 3434,2951,1721,1506,1220,696$. HRMS calcd (found) for $\mathrm{C}_{31} \mathrm{H}_{35} \mathrm{NO}_{2}\left(\mathrm{M}^{+}\right): 453.2668$ (453.2662).

For 4,5-dimethyl-2,3-hexadien-1-ol. TLC (ether- $\left.\mathrm{CH}_{2} \mathrm{Cl}_{2}=1: 30\right): R_{f}=0.43 .{ }^{1} \mathrm{H}$ NMR: $\delta 5.27-$ $5.33(\mathrm{~m}, 1 \mathrm{H}), 4.07(\mathrm{~d}, J=5.6 \mathrm{~Hz}, 2 \mathrm{H}), 2.13$ (d-septet, $J=2.4,6.8 \mathrm{~Hz}, 1 \mathrm{H}), 1.72(\mathrm{~d}, J=2.9 \mathrm{~Hz}, 3 \mathrm{H})$, 1.41 (br s, $1 \mathrm{H}), 1.02(\mathrm{t}, J=6.7 \mathrm{~Hz}, 3 \mathrm{H}), 1.02(\mathrm{~d}, J=6.8 \mathrm{~Hz}, 3 \mathrm{H})$.

For 6,7-dimethyl-2,2-diphenyl-4,5-octadienenitrile. Colorless oil, $80 \%$. TLC (ether- $\mathrm{CH}_{2} \mathrm{Cl}_{2}=$ 1:30): $R_{f}=0.28 .{ }^{1} \mathrm{H}$ NMR: $\delta$ 7.27-7.44 $(\mathrm{m}, 10 \mathrm{H}), 4.99-5.05(\mathrm{~m}, 1 \mathrm{H}), 3.08(\mathrm{~d}, J=7.2 \mathrm{~Hz}, 2 \mathrm{H}), 2.04$ (doublet of sextets, $J=2.1,6.7 \mathrm{~Hz}, 1 \mathrm{H}), 1.56(\mathrm{~d}, J=2.7 \mathrm{~Hz}, 3 \mathrm{H}), 0.95(\mathrm{~d}, J=6.5 \mathrm{~Hz}, 3 \mathrm{H}), 0.93(\mathrm{~d}, J=$ $6.6 \mathrm{~Hz}, 3 \mathrm{H}) .{ }^{13} \mathrm{C}\left\{{ }^{1} \mathrm{H}\right\}$ NMR: $\delta 202.8,140.1,140.0,128.9,128.9,127.9,127.3,127.2,127.2,122.3$, 106.7, 85.1, 52.1, 40.9, 31.9, 21.4, 21.3, 16.8. IR (neat, $\mathrm{cm}^{-1}$ ): 2960, 2867, 1493, 1447, 753, 695 Anal. calcd (found) for $\mathrm{C}_{22} \mathrm{H}_{23} \mathrm{~N}: \mathrm{H}, 7.69$ (7.55); C, 87.66 (87.59).

For 2d. Colorless oil, 58\% (two steps). TLC (EtOAc-hexanes $=1: 9): \quad R_{f}=0.29 . \quad{ }^{1} \mathrm{H}$ NMR $(55$ $\left.{ }^{\circ} \mathrm{C}\right): \quad \delta$ 7.19-7.35 (m, $\left.15 \mathrm{H}\right), 5.04(\mathrm{~s}, 2 \mathrm{H}), 4.73(\mathrm{br} \mathrm{s}, 1 \mathrm{H}), 4.40(\mathrm{br} \mathrm{s}, 1 \mathrm{H}), 4.00(\mathrm{~d}, J=5.8 \mathrm{~Hz}, 2 \mathrm{H})$, 
$2.83(\mathrm{~d}, J=7.5 \mathrm{~Hz}, 2 \mathrm{H}), 2.00$ (doublet of sextets, $J=2.1,6.7 \mathrm{~Hz}, 1 \mathrm{H}$ ), $1.52(\mathrm{~d}, J=2.7 \mathrm{~Hz}, 3 \mathrm{H}$ ), 0.92 $(\mathrm{t}, J=6.8 \mathrm{~Hz}, 6 \mathrm{H}) .{ }^{13} \mathrm{C}\left\{{ }^{1} \mathrm{H}\right\} \operatorname{NMR}\left(55^{\circ} \mathrm{C}\right): \delta 202.3,156.5,145.4,145.3,136.9,128.6,128.4,128.4$, 128.3, 128.2, 128.2, 126.6, 105.3, 86.2, 66.9, 50.8, 48.5, 38.8, 21.6, 21.5, 16.8. IR (neat, $\left.\mathrm{cm}^{-1}\right): 3432$, 2958, 1719, 1505, 1220, 696. Anal. calcd (found) for $\mathrm{C}_{30} \mathrm{H}_{33} \mathrm{NO}_{2}: \mathrm{H}, 7.57$ (7.66); C, 81.97 (81.91).

For 4,5,5-trimethyl-2,3-hexadien-1-ol. ${ }^{9} \quad$ TLC (ether- $\left.\mathrm{CH}_{2} \mathrm{Cl}_{2}=1: 30\right): \quad R_{f}=0.48 .{ }^{1} \mathrm{H}$ NMR: $\delta$ 5.24-5.29 (m, $1 \mathrm{H}), 4.07$ (d, J = 5.6 Hz, $2 \mathrm{H}), 1.72(\mathrm{~d}, J=2.7 \mathrm{~Hz}, 3 \mathrm{H}), 1.40$ (br s, $1 \mathrm{H}), 1.05$ (s, $9 \mathrm{H})$.

For 6,7,7-trimethyl-2,2-diphenyl-4,5-octadienenitrile. Colorless oil, 78\%. TLC (EtOAchexanes = 1:30): $R_{f}=0.30 .{ }^{1} \mathrm{H}$ NMR: $\delta 7.27-7.44(\mathrm{~m}, 10 \mathrm{H}), 4.99(\mathrm{qdd}, J=2.7,6.8,7.7 \mathrm{~Hz}, 1 \mathrm{H}), 3.09$ $(\mathrm{d}, J=6.7 \mathrm{~Hz}, 1 \mathrm{H}), 3.09(\mathrm{~d}, J=7.5 \mathrm{~Hz}, 1 \mathrm{H}), 1.56(\mathrm{~d}, J=1.56 \mathrm{~Hz}, 3 \mathrm{H}), 0.98(\mathrm{~s}, 9 \mathrm{H}) .{ }^{13} \mathrm{C}\left\{{ }^{1} \mathrm{H}\right\} \mathrm{NMR}: \delta$ $202.7,140.1,140.0,128.9,128.9,127.9,127.9,127.3,127.2,122.3,109.6,84.8,52.1,40.9,33.3$ 29.0, 15.0. IR (neat, $\mathrm{cm}^{-1}$ ): 2959, 1959, 1493, 1447, 753, 695. Anal. calcd (found) for $\mathrm{C}_{23} \mathrm{H}_{25} \mathrm{~N}$ : $\mathrm{H}$, 7.99 (8.05); C, 87.57 (87.47).

For 2e. Colorless oil, 38\% over two steps. TLC (EtOAc-hexanes =1:9): $R_{f}=0.29 . \quad{ }^{1} \mathrm{H}$ NMR $\left(55^{\circ} \mathrm{C}\right): \delta \quad 7.18-7.35(\mathrm{~m}, 15 \mathrm{H}), 5.04(\mathrm{~s}, 2 \mathrm{H}), 4.70(\mathrm{br} \mathrm{s}, 1 \mathrm{H}), 4.40(\mathrm{br} \mathrm{s}, 1 \mathrm{H}), 4.00(\mathrm{~d}, J=5.6 \mathrm{~Hz}, 2 \mathrm{H})$, $2.83(\mathrm{~d}, J=7.5 \mathrm{~Hz}, 2 \mathrm{H}), 1.51(\mathrm{~d}, J=2.7 \mathrm{~Hz}, 3 \mathrm{H}), 0.96(\mathrm{~s}, 9 \mathrm{H}) .{ }^{13} \mathrm{C}\left\{{ }^{1} \mathrm{H}\right\} \operatorname{NMR}\left(55^{\circ} \mathrm{C}\right): \delta 202.3,156.5$, $145.4,145.3,136.9,128.6,128.4,128.4,128.4,128.3,128.2,128.2,126.6,108.3,85.8,66.8,50.8$, 48.5, 38.8, 33.4, 29.2, 14.9. IR (neat, $\mathrm{cm}^{-1}$ ): $2957,1720,1504,1448,1220,696$. Anal. calcd (found) for $\mathrm{C}_{31} \mathrm{H}_{35} \mathrm{NO}_{2}: \quad \mathrm{H}, 7.78$ (7.59); C, 82.08 (82.03).

Benzyl (6-ethyl-2,2-diphenyl-4,5-dodecadienyl)carbamate (2f). Ethynyl magnesium bromide (0.5 $\mathrm{M}$ in THF, $100 \mathrm{~mL}, 50.0 \mathrm{mmol})$ was added to a solution of 3-nonanone $(6.70 \mathrm{~mL}, 38.8 \mathrm{mmol})$ in THF/ether $(1: 1,72 \mathrm{~mL})$ at $-10{ }^{\circ} \mathrm{C}$ over 15 minutes. The resulting mixture was warmed to room temperature overnight with stirring, quenched with saturated $\mathrm{NH}_{4} \mathrm{Cl}(100 \mathrm{~mL})$, and extracted with EtOAc $(2 \times 100 \mathrm{~mL})$. The combined organic fractions were washed with brine $(100 \mathrm{~mL})$, dried $\left(\mathrm{MgSO}_{4}\right)$, and concentrated to provide 3-ethyl-1-nonyn-3-ol $(6.37 \mathrm{~g}, 98 \%)$ as a pale yellow solution containing EtOAc 
$(\sim 5 \% \mathrm{w} / \mathrm{w})$ that was used in the subsequent step without further purification. Compound $2 \mathrm{f}$ was synthesized from crude 3-ethyl-1-nonyn-3-ol employing a protocol similar to that used to synthesize $\mathbf{2 a}$.

For 3-ethyl-1-nonyn-3-ol. ${ }^{1} \mathrm{H}$ NMR: $\delta 2.42(\mathrm{~s}, 1 \mathrm{H}), 1.93(\mathrm{br} \mathrm{s}, 1 \mathrm{H}), 1.27-1.71(\mathrm{~m}, 12 \mathrm{H}), 1.03$ (t, $J=7.3 \mathrm{~Hz}, 3 \mathrm{H}), 0.88(\mathrm{t}, J=7.0 \mathrm{~Hz}, 3 \mathrm{H})$.

For 4-ethyl-2,3-decadien-1-ol. TLC (ether- $\left.\mathrm{CH}_{2} \mathrm{Cl}_{2}=1: 30\right): \quad R_{f}=0.48 .{ }^{1} \mathrm{H}$ NMR: $\delta \quad 5.33-5.39$ $(\mathrm{m}, 1 \mathrm{H}), 4.07(\mathrm{~d}, J=5.6 \mathrm{~Hz}, 2 \mathrm{H}), 1.94-2.00(\mathrm{~m}, 4 \mathrm{H}), 1.23-1.45(\mathrm{~m}, 9 \mathrm{H}), 0.99(\mathrm{t}, J=7.3 \mathrm{~Hz}, 3 \mathrm{H}), 0.87$ (t, $J=7.0 \mathrm{~Hz}, 3 \mathrm{H})$.

For 6-ethyl-2,2-diphenyl-4,5-dodecadienenitrile. Colorless oil, 85\%. TLC $($ EtOAc-hexanes $=$ 1:19): $R_{f}=0.36 .{ }^{1} \mathrm{H}$ NMR (Figure S4): $\delta 7.27(\mathrm{~m}, 10 \mathrm{H}), 5.04-5.11(\mathrm{~m}, 1 \mathrm{H}), 3.09(\mathrm{~d}, \mathrm{~J}=7.2 \mathrm{~Hz}, 2 \mathrm{H})$, 1.78-1.92 (m, $4 \mathrm{H}), 1.22-1.34(\mathrm{~m}, 8 \mathrm{H}), 0.90(\mathrm{t}, J=6.8 \mathrm{~Hz}, 3 \mathrm{H}), 0.89(\mathrm{t}, J=7.3 \mathrm{~Hz}, 3 \mathrm{H}) .{ }^{13} \mathrm{C}\left\{{ }^{1} \mathrm{H}\right\} \mathrm{NMR}$ (Figure S5): $\delta$ 203.0, 140.1, 128.9, 127.9, 127.3, 122.3, 107.3, 86.2, 52.0, 40.9, 32.4, 31.8, 29.2, 27.6, 25.5, 22.8, 14.3, 12.3. IR (neat, $\mathrm{cm}^{-1}$ ): 2924, 2854, 1493, 1449, 753, 695. HRMS calcd (found) for $\mathrm{C}_{26} \mathrm{H}_{31} \mathrm{~N}\left(\mathrm{M}^{+}\right): 357.2456$ (357.2453).

For 2f. Colorless oil, $50 \%$ (two steps). TLC $\left(\mathrm{CH}_{2} \mathrm{Cl}_{2}\right.$-hexanes $\left.=1: 1\right): \quad R_{f}=0.29 .{ }^{1} \mathrm{H}$ NMR (55 ${ }^{\circ} \mathrm{C}, 300 \mathrm{MHz}$, Figure S6): $\delta$ 7.18-7.33 (m, $\left.15 \mathrm{H}\right), 5.03(\mathrm{~s}, 2 \mathrm{H}), 4.77$ (br s, $\left.1 \mathrm{H}\right), 4.40$ (br s, $\left.1 \mathrm{H}\right), 4.00$ $(\mathrm{d}, J=5.9 \mathrm{~Hz}, 2 \mathrm{H}), 2.84(\mathrm{~d}, J=7.4 \mathrm{~Hz}, 2 \mathrm{H}), 1.76-1.88(\mathrm{~m}, 4 \mathrm{H}), 1.22-1.33(\mathrm{~m}, 8 \mathrm{H}), 0.90(\mathrm{t}, J=6.7 \mathrm{~Hz}$, $3 \mathrm{H}), 0.88(\mathrm{t}, J=7.3 \mathrm{~Hz}, 3 \mathrm{H}) .{ }^{13} \mathrm{C}\left\{{ }^{1} \mathrm{H}\right\} \mathrm{NMR}\left(55^{\circ} \mathrm{C}, 75 \mathrm{MHz}\right.$, Figure S7): $\delta 202.6,156.5,145.4,145.3$, 128.6, 128.4, 128.4, 128.2, 128.2, 126.6, 126.6, 105, 9, 87.2, 66.9, 50.9, 48.6, 39.0, 32.6, 31.9, 29.2, 27.9, 25.7, 22.8, 14.2, 12.5. IR (neat, $\mathrm{cm}^{-1}$ ): 2925, 1721, 1506, 1449, 1220, 696. HRMS calcd (found) for $\mathrm{C}_{34} \mathrm{H}_{41} \mathrm{NO}_{2}\left(\mathrm{M}^{+}\right): 495.3137$ (495.3142).

Benzyl (6-methyl-4,5-dodecadienyl)carbamate (2g). A solution of 3-methyl-nonyn-3-ol (15.43 $\mathrm{g}, 100.0 \mathrm{mmol})$, triethylorthoacetate $(55 \mathrm{~mL}, 300 \mathrm{mmol})$, and propionic acid $(0.4 \mathrm{~mL}, 5.3 \mathrm{mmol})$ was heated at reflux overnight with azeotropic removal of ethanol. The reaction mixture was cooled to room temperature, diluted with ether $(100 \mathrm{~mL})$, washed with $1 \mathrm{M} \mathrm{HCl}(50 \mathrm{~mL})$, saturated $\mathrm{NaHCO}_{3}(50 \mathrm{~mL})$, 
and brine $(50 \mathrm{~mL})$, dried $\left(\mathrm{MgSO}_{4}\right)$, and concentrated. The resulting oil was chromatographed $\left(\mathrm{CH}_{2} \mathrm{Cl}_{2}\right)$ to provide ethyl 5-methyl-3,4-undecadienoate $(\mathbf{S 7}, 8.09 \mathrm{~g}, 35 \%)$ as a yellow oil.

S7 (5.97 g, $26.4 \mathrm{mmol})$ was added to a stirred suspension of $\mathrm{LiAlH}_{4}(5.19 \mathrm{~g}, 137 \mathrm{mmol})$ in ether $(200 \mathrm{~mL})$ at $0{ }^{\circ} \mathrm{C}$ and warmed to room temperature overnight. The reaction mixture was cooled to $0{ }^{\circ} \mathrm{C}$ and quenched by successive addition of water $(7.2 \mathrm{~mL}), 15 \% \mathrm{NaOH}[(\mathrm{w} / \mathrm{w}), 7.2 \mathrm{~mL}]$ and water $(7.2$ $\mathrm{mL})$. The resulting white suspension was filtered through Celite, eluted with ether (200 $\mathrm{mL})$, and concentrated. The resulting residue was concentrated to provide 5-methyl-3,4-undecadien-1-ol (S8, $5.98 \mathrm{~g}, 95 \%)$ as a yellow oil that was used without further purification.

A solution of $\mathrm{TsCl}(2.38 \mathrm{~g}, 12.5 \mathrm{mmol})$ in $\mathrm{CH}_{2} \mathrm{Cl}_{2}(10 \mathrm{~mL})$ was added to a solution of $\mathbf{S 8}(1.81 \mathrm{~g}$, $9.95 \mathrm{mmol})$, DMAP (120 mg, $0.98 \mathrm{mmol})$, and triethylamine $(2.1 \mathrm{~mL}, 15 \mathrm{mmol})$ in $\mathrm{CH}_{2} \mathrm{Cl}_{2}(35 \mathrm{~mL})$ at 0 ${ }^{\circ} \mathrm{C}$ and the reaction maintained at $0{ }^{\circ} \mathrm{C}$ overnight. The resulting suspension was treated with water (50 $\mathrm{mL})$, the layers were separated and the aqueous layer was extracted with $\mathrm{CH}_{2} \mathrm{Cl}_{2}(2 \times 25 \mathrm{~mL})$. The combined organic extracts were washed with water $(25 \mathrm{~mL}), 1 \mathrm{M} \mathrm{HCl}(25 \mathrm{~mL})$, saturated $\mathrm{NaHCO}_{3}(25$ $\mathrm{mL})$, and brine $(25 \mathrm{~mL})$, dried $\left(\mathrm{MgSO}_{4}\right)$, and concentrated. The resulting yellow oil was chromatographed $\left(\mathrm{CH}_{2} \mathrm{Cl}_{2}\right.$-hexanes $\left.=1: 2\right)$ to provide 5-methyl-3,4-undecadienyl-toluenesulfonate (S9) which was concentrated to $\sim 3 \mathrm{~mL}$ and used immediately in the subsequent step.

$\mathrm{KCN}$ (781 mg, $12.0 \mathrm{mmol}$ ) was added to a solution of $\mathbf{S 9}$ (all material obtained in previous step) in DMF $(25 \mathrm{~mL})$ and the reaction mixture was stirred vigorously at room temperature for 3 days. The resulting suspension was partitioned between ether and water $(1: 1,200 \mathrm{~mL})$, the layers were deparated, and the organic fraction was dried $\left(\mathrm{MgSO}_{4}\right)$ and concentrated. The resulting oil was chromatographed $($ EtOAc-hexanes $=1: 19)$ to give 6-methyl-4,5-dodecadienenitrile (S10, 968 mg, 51 \%) as a colorless oil. Compound $\mathbf{2} \mathbf{g}$ was prepared from $\mathbf{S 1 0}$ by a manner analogous to that used to prepare $\mathbf{2 a}$ from $\mathbf{S 5 .}$

For S7. $\operatorname{TLC}\left(\mathrm{CH}_{2} \mathrm{Cl}_{2}\right): R_{f}=0.72 .{ }^{1} \mathrm{H} \mathrm{NMR}: \delta \quad 5.09-5.16(\mathrm{~m}, 1 \mathrm{H}), 4.14(\mathrm{q}, J=7.2 \mathrm{~Hz}, 2 \mathrm{H})$, $2.96(\mathrm{~d}, J=7.2 \mathrm{~Hz}, 2 \mathrm{H}), 1.92(\mathrm{dt}, J=2.7,7.9 \mathrm{~Hz}, 2 \mathrm{H}), 1.66(\mathrm{~d}, J=2.9 \mathrm{~Hz}, 3 \mathrm{H}), 1.24-1.41(\mathrm{~m}, 11 \mathrm{H})$, $0.87(\mathrm{t}, J=6.8 \mathrm{~Hz}, 3 \mathrm{H})$. 
For S8. ${ }^{1} \mathrm{H}$ NMR: $\delta$ 4.97-5.03 $(\mathrm{m}, 1 \mathrm{H}), 3.65-3.69(\mathrm{~m}, 2 \mathrm{H}), 2.18-2.23(\mathrm{~m}, 2 \mathrm{H}), 1.90-1.94(\mathrm{~m}, 2$ $\mathrm{H}), 1.72(\mathrm{br} \mathrm{s}, 1 \mathrm{H}), 1.24-1.42(\mathrm{~m}, 8 \mathrm{H}), 0.87(\mathrm{t}, J=6.8 \mathrm{~Hz}, 3 \mathrm{H})$.

For S10. TLC (EtOAc-hexanes $=1: 19): R_{f}=0.29 .{ }^{1} \mathrm{H}$ NMR (Figure S8): $\delta 5.05-5.12(\mathrm{~m}, 1 \mathrm{H})$, 2.40 (ddd, $J=1.2,6.8,7.5,2 \mathrm{H}), 2.27-2.32(\mathrm{~m}, 2 \mathrm{H}), 1.93-1.98(\mathrm{~m}, 2 \mathrm{H}), 2.90(\mathrm{~d}, J=2.90 \mathrm{~Hz}, 3 \mathrm{H})$, 1.25-1.42 (m, $8 \mathrm{H}), 0.88(\mathrm{t}, J=6.8 \mathrm{~Hz}, 6 \mathrm{H}) . \quad{ }^{13} \mathrm{C}\left\{{ }^{1} \mathrm{H}\right\}$ NMR (Figure S9): $\delta \quad 201.5,119.7,102.9,87.2$, $34.1,31.9,29.1,27.6,25.2,22.8,19.2,16.8,14.2$. IR (neat, $\mathrm{cm}^{-1}$ ): 2924, 2855, 2245, 1445, 1371, 1180. HRMS calcd (found) for $\mathrm{C}_{13} \mathrm{H}_{21} \mathrm{~N}^{+}\left(\mathrm{M}^{+}\right)$: 191.1674 (191.1675).

For 2g. Colorless oil, 49\% (two steps). TLC (EtOAc-hexanes $=1: 9): \quad R_{f}=0.24 .{ }^{1} \mathrm{H}$ NMR (55 $\left.{ }^{\circ} \mathrm{C}\right): \delta$ 7.28-7.37 (m, $\left.5 \mathrm{H}\right), 5.11(\mathrm{~s}, 2 \mathrm{H}), 4.97-5.02(\mathrm{~m}, 1 \mathrm{H}), 4.72(\mathrm{br} \mathrm{s}, 1 \mathrm{H}), 3.24(\mathrm{dt}, J=6.3,6.8 \mathrm{~Hz}, 2$ H), 1.97-2.03 (m, $2 \mathrm{H}), 1.93(\mathrm{dt}, J=2.9,7.9 \mathrm{~Hz}, 2 \mathrm{H}), 1.67$ (d, J = 2.9 Hz, $3 \mathrm{H}), 1.61$ (septet, $J=7.2 \mathrm{~Hz}$, $2 \mathrm{H}), 1.25-1.45(\mathrm{~m}, 8 \mathrm{H}), 0.90(\mathrm{t}, J=7.0 \mathrm{~Hz}, 3 \mathrm{H}) .{ }^{13} \mathrm{C}\left\{{ }^{1} \mathrm{H}\right\} \operatorname{NMR}\left(55^{\circ} \mathrm{C}\right): \delta 201.7,156.5,137.0,128.6$, 128.2, 100.2, 89.2, 66.8, 40.9, 34.3, 31.9, 29.6, 29.1, 27.8, 26.7, 22.8, 19.3, 14.1. IR (neat, $\mathrm{cm}^{-1}$ ): 3335, 2925, 2856, 1699, 1525, 1246. Anal. calcd (found) for $\mathrm{C}_{21} \mathrm{H}_{31} \mathrm{NO}_{2}: \mathrm{H}, 9.48(9.46) ; \mathrm{C}, 76.55$ (76.44).

\section{Pyrrolidines}

Benzyl 2-(2-methyl-1-butenyl)-4,4-diphenylpyrrolidine-1-carboxylate (3a). A mixture of $(S)$ $1\left(6.1 \mathrm{mg}, 3.8 \times 10^{-3} \mathrm{mmol}\right)$ and $\mathrm{AgClO}_{4}\left(1.6 \mathrm{mg}, 7.5 \times 10^{-3} \mathrm{mmol}\right)$ in $\mathrm{m}$-xylene $(0.2 \mathrm{~mL})$ was stirred at room temperature for $5 \mathrm{~min}$, treated with a solution of $2 \mathrm{a}(63.8 \mathrm{mg}, 0.15 \mathrm{mmol})$ in $m$-xylene $(0.3 \mathrm{~mL})$ and stirred at room temperature for $24 \mathrm{~h}$. Column chromatography of the crude reaction mixture $\left(\mathrm{CH}_{2} \mathrm{Cl}_{2}\right.$-hexanes $\left.=1: 1 \rightarrow 10: 1\right)$ gave $3 \mathrm{a}(60.0 \mathrm{mg}, 94 \%)$ as a colorless oil comprised of a $3.1: 1$ mixture of $(R, Z)-3 a\left(95 \%\right.$ ee) and $(R, E)-3 a\left(72 \%\right.$ ee). Anal. calcd (found) for $\mathrm{C}_{29} \mathrm{H}_{31} \mathrm{NO}_{2}: \mathrm{H}, 7.34(7.27) ; \mathrm{C}$, 81.85 (81.81). IR (neat, $\mathrm{cm}^{-1}$ ): 2968, 2872, 1696, 1410, 1104, 696.

The enantiomeric purities of $(R, E)-3 \mathbf{a}$ and $(R, Z)-3 \mathbf{a}$ were determined by HPLC analysis (Figure S10). Determination of the absolute configuration of $(R, E)-3 \mathbf{a}$ and $(R, Z)-3 \mathbf{a}$ is described below. The 
purified 3.1:1 mixture of $(R, Z)-3 \mathbf{a}$ and $(R, E)$-3a was further chromatographed $\left(\mathrm{CH}_{2} \mathrm{Cl}_{2}\right.$-hexanes $=1: 1 \rightarrow$ $10: 1)$ to give a 9:1 mixture of $(R, Z)-3 \mathbf{a}$ and $(R, E)-3 \mathbf{a}$. A racemic 7.3:1 sample of $(E)-\mathbf{3 a}$ and $(Z)-\mathbf{3 a}$ was obtained from reaction of $2 \mathrm{a}$ with $\mathrm{Au}\left[\mathrm{P}(t-\mathrm{Bu})_{2}(o-\right.$ biphenyl) $] \mathrm{Cl} / \mathrm{AgOTf}$. The relative stereochemistry of $(E)-3 a$ and (Z)-3a were unambiguously assigned through independent nOe analysis of samples enriched in each diastereomer (Figures S11 and S12).

For (Z)-3a: $\operatorname{TLC}\left(\mathrm{CH}_{2} \mathrm{Cl}_{2}\right.$-hexanes $\left.=5: 1\right): R_{f}=0.45 .{ }^{1} \mathrm{H}$ NMR $(500 \mathrm{MHz}, 1.4: 1$ mixture of rotamers): $\delta$ 7.14-7.37 (m, $15 \mathrm{H}),[5.28(\mathrm{~d}, J=7.5 \mathrm{~Hz}), 5.15(\mathrm{~d}, J=7.5 \mathrm{~Hz}), 1.4: 1,1 \mathrm{H}], 5.00-5.04(\mathrm{~m}, 2$ H), [4.72 (d, J = 11.0 Hz), $4.59(\mathrm{~d}, J=12.0 \mathrm{~Hz}), 1.4: 1,1 \mathrm{H}],[4.42(\mathrm{dt}, J=7.5,8.5 \mathrm{~Hz}), 4.30(\mathrm{dt}, J=7.0$, $9.0 \mathrm{~Hz}), 1: 1.4,1 \mathrm{H}],[3.67(\mathrm{~d}, J=12.0 \mathrm{~Hz}), 3.64(\mathrm{~d}, J=11.5 \mathrm{~Hz}), 1: 1.4,1 \mathrm{H}], 2.72-2.79(\mathrm{~m}, 1 \mathrm{H}),[2.36$ (dd, $J=10.0,12.5 \mathrm{~Hz}), 2.31(\mathrm{dd}, J=10.0,12.5 \mathrm{~Hz}), 1.4: 1,1 \mathrm{H}], 2.14-2.21(\mathrm{~m}, 1 \mathrm{H}), 2.14-2.21(\mathrm{~m}, 0.4$ $\mathrm{H}), 1.85-2.03(\mathrm{~m}, 1.2 \mathrm{H}), 1.65-1.72(\mathrm{~m}, 0.4 \mathrm{H}),[1.68(\mathrm{~s}), 1.61(\mathrm{~s}), 1: 1.4,3 \mathrm{H}],[0.98(\mathrm{t}, J=7.5 \mathrm{~Hz}), 0.79$ $(\mathrm{t}, J=7.5 \mathrm{~Hz}), 1: 1.4,3 \mathrm{H}] .{ }^{13} \mathrm{C}\left\{{ }^{1} \mathrm{H}\right\} \mathrm{NMR}(126 \mathrm{MHz}, 1.4: 1$ mixture of rotamers): $\delta 155.5,154.7,145.9$ $145.2,139.5,138.4,137.3,137.2,128.9,128.7,128.5,128.2,127.8,127.0,126.7,126.7,126.6,126.5$, $126.5,125.7,66.9,66.9,56.1,54.8,54.3,53.2,52.7,46.1,45.4,25.3,25.0,23.1,22.9,13.3,13.0$.

For (E)-3a: TLC $\left(\mathrm{CH}_{2} \mathrm{Cl}_{2}-\right.$ hexanes $\left.=5: 1\right): \quad R_{f}=0.39 .{ }^{1} \mathrm{H}$ NMR $(500 \mathrm{MHz}, 1.7: 1$ mixture of rotamers): $\delta$ 7.15-7.37 (m, $15 \mathrm{H}),[5.28(\mathrm{~d}, J=12.4 \mathrm{~Hz}), 5.14(\mathrm{~d}, J=12.8 \mathrm{~Hz}), 1: 1.7,1 \mathrm{H}], 4.99-5.05$ $(\mathrm{m}, 2 \mathrm{H}),[4.71(\mathrm{dd}, J=2.0,11.6 \mathrm{~Hz}), 4.56(\mathrm{~d}, J=10.4 \mathrm{~Hz}), 1.7: 1,1 \mathrm{H}],[4.43(\mathrm{dt}, J=6.8,8.8 \mathrm{~Hz}), 4.31$ (dt, $J=7.2,8.8 \mathrm{~Hz}), 1: 1.7,1 \mathrm{H}],[3.72(\mathrm{~d}, J=12.0 \mathrm{~Hz}), 3.69(\mathrm{~d}, J=11.6 \mathrm{~Hz}), 1: 1.7,1 \mathrm{H}], 2.75-2.83(\mathrm{~m}, 1$ $\mathrm{H}),[2.35(\mathrm{dd}, J=9.6,12.0 \mathrm{~Hz}), 2.30(\mathrm{dd}, J=10.4,12.8 \mathrm{~Hz}), 1.7: 1,1 \mathrm{H}], 1.85-2.01(\mathrm{~m}, 1.6 \mathrm{H}), 1.66-1.71$ $(\mathrm{m}, 0.4 \mathrm{H}),[1.66(\mathrm{~s}), 1.38(\mathrm{~s}), 1: 1.7,3 \mathrm{H}],[0.94(\mathrm{t}, J=7.2 \mathrm{~Hz}), 0.88(\mathrm{~d}, J=7.2 \mathrm{~Hz}), 1: 1.7,3 \mathrm{H}] .{ }^{13} \mathrm{C}\left\{{ }^{1} \mathrm{H}\right\}$ NMR (126 MHz, 1.7:1 mixture of rotamers): $\delta 155.6,145.9,145.2,139.2,138.4,137.3,137.1,128.8$, $128.7,128.5,128.2,127.8,127.0,126.7,125.0,124.4,67.0,66.9,56.1,55.2,54.6,53.2,52.7,45.8$, $45.1,32.3,32.2,16.7,16.4,12.6,12.4$

All remaining enantioselective hydroamination reactions were performed employing a procedure analogous to that used to synthesize $\mathbf{3 a}$ from $\mathbf{2 a}$. For all remaining pyrrolidines, samples 
enriched in the (Z)-diastereomer were obtained from the enantioselective reaction. Samples enriched in the $(E)$-diastereomer were obtained from reaction of the appropriate $N$-allenyl carbamate with $\operatorname{Au}[\mathrm{P}(t-$ $\mathrm{Bu})_{2}$ (o-biphenyl)]Cl/AgOTf. Chiral HPLC analyses for enantiomerically enriched and racemic pyrrolidines are depicted in Figures S13-S18. The stereochemistry of all pyrrolidines were assigned and verified by nOe analysis.

Benzyl 2-(2-methyl-1-octenyl)-4,4-diphenylpyrrolidine-1-carboxylate (3b). Colorless oil. Anal. calcd (found) for $\mathrm{C}_{33} \mathrm{H}_{39} \mathrm{NO}_{2}: \mathrm{H}, 8.16$ (8.19); C, 82.29 (82.24). IR (neat, $\mathrm{cm}^{-1}$ ): 2925, 1698, $1410,1351,697$. NMR analysis was performed on an enantiomerically enriched 32:1 mixture of (Z)$3 \mathbf{b} /(E)-3 \mathbf{b}$ and on a racemic $\geq 50: 1$ mixture of $(E)-3 \mathbf{b} /(Z)-3 \mathbf{b}$.

For (Z)-3b: $\operatorname{TLC}\left(\mathrm{CH}_{2} \mathrm{Cl}_{2}\right.$-hexanes $\left.=5: 1\right): R_{f}=0.52 .{ }^{1} \mathrm{H}$ NMR (1.6:1 mixture of rotamers): $\delta$ 7.15-7.37 (m, $15 \mathrm{H}),[5.28(\mathrm{~d}, J=12.8 \mathrm{~Hz}), 5.15(\mathrm{~d}, J=12.4 \mathrm{~Hz}), 1: 1.6,1 \mathrm{H}], 5.00-5.07(\mathrm{~m}, 2 \mathrm{H}),[4.73$ (dd, $J=1.6,11.6 \mathrm{~Hz}), 4.59(\mathrm{~d}, J=11.2 \mathrm{~Hz}), 1.6: 1,1 \mathrm{H}],[4.41(\mathrm{dt}, J=7.6,8.4 \mathrm{~Hz}), 4.29(\mathrm{dt}, J=6.8,9.2$ $\mathrm{Hz}), 1: 1.6,1 \mathrm{H}],[3.69(\mathrm{~d}, J=11.2 \mathrm{~Hz}), 3.67(\mathrm{~m}, J=11.6 \mathrm{~Hz}), 1: 1.6,1 \mathrm{H}], 2.72-2.80(\mathrm{~m}, 1 \mathrm{H}),[2.36(\mathrm{dd}$, $J=10.0,12.4 \mathrm{~Hz}), 2.31(\mathrm{dd}, J=9.6,12.8 \mathrm{~Hz}), 1.6: 1,1 \mathrm{H}], 2.20-2.29(\mathrm{~m}, 0.4 \mathrm{H})$, 1.84-1.99 $(\mathrm{m}, 1 \mathrm{H})$, [1.69 (s), 1.61 (s), 1:1.6, $3 \mathrm{H}], 1.54-1.66(\mathrm{~m}, 0.6 \mathrm{H}), 1.05-1.47(\mathrm{~m}, 9 \mathrm{H}),[0.86(\mathrm{t}, J=6.0 \mathrm{~Hz}), 0.83(\mathrm{t}, \mathrm{J}=$ 7.2), $3 \mathrm{H}] . \quad{ }^{13} \mathrm{C}\left\{{ }^{1} \mathrm{H}\right\}$ NMR (126 MHz, $1.6: 1$ mixture of rotamers): $\delta \quad 155.5,154.7,145.8,145.1,138.3$, $137.3,137.2,137.1,128.8,128.7,128.4,128.2,127.8,128.8,128.7,128.4,128.2,127.8,127.0,126.7$ 126.6, 126.6, 126.2, 66.9, 66.8, 56.2, 54.9, 54.4, 53.2, 52.7, 46.0, 45.3, 32.2, 32.0, 31.8, 29.4, 29.4, $28.4,28.0,23.7,23.5,22.8,22.8,14.3$.

For (E)-3b: $\operatorname{TLC}\left(\mathrm{CH}_{2} \mathrm{Cl}_{2}\right.$-hexanes $\left.=5: 1\right): \quad R_{f}=0.52 .{ }^{1} \mathrm{H}$ NMR (1.7:1 mixture of rotamers): $\delta$ 7.15-7.37 (m, $15 \mathrm{H}),[5.27(\mathrm{~d}, J=12.0 \mathrm{~Hz}), 5.11(\mathrm{~d}, J=12.4), 1: 1.7,1 \mathrm{H}], 5.01-5.06(\mathrm{~m}, 2 \mathrm{H}),[4.70(\mathrm{dd}$, $J=2.0,11.6 \mathrm{~Hz}), 4.55(\mathrm{~d}, J=11.2 \mathrm{~Hz}), 1.7: 1,1 \mathrm{H}],[4.41(\mathrm{dt}, J=7.2,8.8 \mathrm{~Hz}), 4.30(\mathrm{dt}, J=6.8,9.2 \mathrm{~Hz})$, 1:1.7, $1 \mathrm{H}],[3.71(\mathrm{~d}, J=12.0 \mathrm{~Hz}), 3.68(\mathrm{~d}, J=11.6 \mathrm{~Hz}), 1: 1.7,1 \mathrm{H}], 2.74-2.83(\mathrm{~m}, 1 \mathrm{H}),[2.35(\mathrm{dd}, J=$ 10.0, $12.8 \mathrm{~Hz}), 2.29(\mathrm{dd}, J=10.0,12.8 \mathrm{~Hz}), 1.7: 1,1 \mathrm{H}], 1.83-1.96(\mathrm{~m}, 2 \mathrm{H}),[1.64(\mathrm{~s}), 1.38(\mathrm{~s}), 1: 1.7,3$ $\mathrm{H}], 1.18-1.30(\mathrm{~m}, 8 \mathrm{H}), 0.87(\mathrm{t}, J=7.2,3 \mathrm{H}) .{ }^{13} \mathrm{C}\left\{{ }^{1} \mathrm{H}\right\}$ NMR $(126 \mathrm{MHz}, 1.7: 1$ mixture of rotamers): $\delta$ 
$155.6,152.7,145.9,145.2,137.9,137.1,137.0,128.2,128.7,128.5,128.2,127.9,127.8,127.0,126.7$ $126.7,126.6,126.5,126.1,125.5,67.0,66.8,56.1,55.2,54.6,53.2,52.8,45.8,45.0,39.7,39.6,32.0$, $29.2,27.9,27.8,22.8,16.7,16.4,14.3$.

Benzyl 2-(2,4-dimethyl-1-pentenyl)-4,4-diphenylpyrrolidine-1-carboxylate (3c). Colorless oil. Anal. calcd (found) for $\mathrm{C}_{31} \mathrm{H}_{35} \mathrm{NO}_{2}$ : H, 7.78 (7.75); C, 82.08 (81.96). IR (neat, $\mathrm{cm}^{-1}$ ): 2953, 1697, $1409,1351,1100,696$. NMR analysis was performed on an enantiomerically enriched $\geq 50: 1$ mixture of $(Z)-3 c /(E)-3 c$ and on a racemic $\geq 50: 1$ mixture of $(E)-3 c /(Z)-3 c$.

For (Z)-3c: TLC (hexanes-EtOAc $=5: 1): \quad R_{f}=0.42 . \quad{ }^{1} \mathrm{H}$ NMR $(500 \mathrm{MHz}, 1.6: 1$ mixture of rotamers): $\delta$ 7.19-7.38 $(\mathrm{m}, 15 \mathrm{H}),[5.29(\mathrm{~d}, J=12.5 \mathrm{~Hz}), 5.16(\mathrm{~d}, J=12.5 \mathrm{~Hz}), 1: 1.6,1 \mathrm{H}], 5.12(\mathrm{~d}, J=$ 8.0 Hz, $1 \mathrm{H}),[5.05(\mathrm{~d}, J=12.5 \mathrm{~Hz}), 5.01(\mathrm{~d}, J=12.5 \mathrm{~Hz}), 1: 1.6,1 \mathrm{H}],[4.73(\mathrm{~d}, J=11.5 \mathrm{~Hz}), 4.60(\mathrm{~d}, J=$ $11.0 \mathrm{~Hz}), 1.6: 1,1 \mathrm{H}],[4.45(\mathrm{dt}, J=7.5,8.5 \mathrm{~Hz}), 4.31(\mathrm{dt}, J=7.5,8.5 \mathrm{~Hz}), 1: 1.6,1 \mathrm{H}],[3.37(\mathrm{~d}, J=12.0$ $\mathrm{Hz}), 3.70(\mathrm{~d}, J=11.5 \mathrm{~Hz}), 1: 1.6,1 \mathrm{H}], 2.77-2.85(\mathrm{~m}, 1 \mathrm{H}), 2.30-2.39(\mathrm{~m}, 1 \mathrm{H}), 2.19(\mathrm{dd}, J=10.0,12.5$ $\mathrm{Hz}, 0.4 \mathrm{H}), 1.39-1.89(\mathrm{~m}, 5.6 \mathrm{H}),[0.93(\mathrm{~d}, J=6.5 \mathrm{~Hz}), 0.71(\mathrm{~d}, J=6.5 \mathrm{~Hz}), 1: 1.6,3 \mathrm{H}],[0.80(\mathrm{~d}, J=6.0)$, $0.76(\mathrm{~d}, J=6.0 \mathrm{~Hz}), 1: 1.6,3 \mathrm{H}] .{ }^{13} \mathrm{C}\left\{{ }^{1} \mathrm{H}\right\} \mathrm{NMR}(126 \mathrm{MHz}, 1.6: 1$ mixture of rotamers): $\delta \quad 155.5,154.6$, $145.8,145.0,137.2,137.1,136.2,128.8,128.6,128.4,128.0,127.9,127.8,127.2,126.9,126.6,126.5$, $126.5,66.9,66.8,56.2,54.8,54.3,53.1,52.6,45.9,45.1,41.4,41.3,26.7,26.4,24.0,23.7,23.3,23.1$, 22.2 .

For $(E)-3 \mathrm{c}:$ TLC (hexanes-EtOAc $=5: 1): \quad R_{f}=0.46 .{ }^{1} \mathrm{H}$ NMR $(500 \mathrm{MHz}, 1.7: 1$ mixture of rotamers): $\delta$ 7.19-7.40 (m, $15 \mathrm{H}),[5.29(\mathrm{~d}, J=12.5 \mathrm{~Hz}), 5.15(\mathrm{~d}, J=12.5 \mathrm{~Hz}), 1: 1.7,1 \mathrm{H}], 5.04-5.10$ $(\mathrm{m}, 2 \mathrm{H}),[4.75(\mathrm{~d}, J=12.0 \mathrm{~Hz}), 4.59(\mathrm{~d}, J=11.5 \mathrm{~Hz}), 1.7: 1,1 \mathrm{H}],[4.45(\mathrm{dt}, J=7.5,8.5 \mathrm{~Hz}), 4.34(\mathrm{dt}, J=$ 7.0, 8.5 Hz), 1:1.7, $1 \mathrm{H}],[3.75(\mathrm{~d}, J=12.0 \mathrm{~Hz}), 3.72(\mathrm{~d}, J=12.0 \mathrm{~Hz}), 1: 1.7,1 \mathrm{H}], 2.80-2.87(\mathrm{~m}, 1 \mathrm{H})$, [2.39 (dd, $J=10.0,12.5 \mathrm{~Hz}), 2.33(\mathrm{dd}, J=10.0,12.0), 1.7: 1,1 \mathrm{H}], 1.60-1.91(\mathrm{~m}, 3 \mathrm{H}),[1.66$ (s), 1.39 (s), 1:1.7, $3 \mathrm{H}], 0.87(\mathrm{~d}, J=6.5 \mathrm{~Hz}, 1.2 \mathrm{H}), 0.79-0.81(\mathrm{~m}, 4.8 \mathrm{H}) .{ }^{13} \mathrm{C}\left\{{ }^{1} \mathrm{H}\right\}$ NMR $(126 \mathrm{MHz}, 1.7: 1$ mixture of rotamers): $\delta 155.5,154.5,145.8,145.1,137.3,137.1,136.6,135.7,128.7,128.6,128.6,128.4$, 
128.1, 128.1, 127.8, 127.6, 127.0, 126.9, 126.6, 126.6, 126.5, 126.4, 66.9, 66.7, 56.1, 55.1, 54.6, 53.1, $52.7,49.6,49.4,45.7,44.9$.

Benzyl 2-(2,3-dimethyl-1-butenyl)-4,4-diphenylpyrrolidine-1-carboxylate (3d). Colorless oil. Anal. calcd (found) for $\mathrm{C}_{30} \mathrm{H}_{33} \mathrm{NO}_{2}$ : $\mathrm{H}, 7.57$ (7.53); C, 81.97 (81.79). IR (neat, $\mathrm{cm}^{-1}$ ): 2960, 2872, $1700,1412,1354,696$. NMR analysis was performed on an enantiomerically enriched $\geq 50: 1$ mixture of $(Z)-\mathbf{3 d} /(E)-\mathbf{3 d}$ and on a racemic $24: 1$ mixture of $(E)-3 \mathbf{d} /(Z)-3 \mathbf{d}$.

For (Z)-3d: $\operatorname{TLC}\left(\mathrm{CH}_{2} \mathrm{Cl}_{2}\right.$-hexanes $\left.=1: 1\right): R_{f}=0.18 .{ }^{1} \mathrm{H}$ NMR $(500 \mathrm{MHz}, 1.6: 1$ mixture of rotamers): $\delta$ 7.15-7.34 $(\mathrm{m}, 15 \mathrm{H}),[5.26(\mathrm{~d}, J=12.5 \mathrm{~Hz}), 5.12(\mathrm{~d}, J=13.0 \mathrm{~Hz}), 1: 1.6,1 \mathrm{H}],[5.04(\mathrm{~d}, J=$ $12.5 \mathrm{~Hz}), 5.02(\mathrm{~d}, J=12.5 \mathrm{~Hz}), 1.6: 1,1 \mathrm{H}], 4.98(\mathrm{~d}, J=8.5 \mathrm{~Hz}, 1 \mathrm{H}),[4.71(\mathrm{dd}, J=1.5,11.5 \mathrm{~Hz}), 4.57$ $(\mathrm{d}, J=11.5 \mathrm{~Hz}), 1.6: 1,1 \mathrm{H}],[4.49(\mathrm{dt}, J=7.5,8.5 \mathrm{~Hz}), 4.38(\mathrm{dt}, J=7.0,9.0 \mathrm{~Hz}), 1: 1.6,1 \mathrm{H}],[3.65(\mathrm{~d}, J$ $=13.5 \mathrm{~Hz}$ ), $3.63(\mathrm{~d}, J=11.5 \mathrm{~Hz}), 1: 1.6,1 \mathrm{H}],[2.80$ (septet, $J=6.5 \mathrm{~Hz}$ ), 2.58 (septet, $J=7.0 \mathrm{~Hz}), 1: 1.6$, $1 \mathrm{H}], 2.69-2.77(\mathrm{~m}, 1 \mathrm{H}),[2.34(\mathrm{dd}, J=10.0,12.5 \mathrm{~Hz}), 2.29(\mathrm{dd}, J=9.5,12.5 \mathrm{~Hz}), 1.6: 1,1 \mathrm{H}],[1.58$ (s), 1.52 (s), 1:1.6, $3 \mathrm{H}],[0.99(\mathrm{~d}, J=7.0 \mathrm{~Hz}), 0.86(\mathrm{~d}, J=7.0 \mathrm{~Hz}), 1: 1.6,3 \mathrm{H}],[0.92(\mathrm{~d}, J=7.0 \mathrm{~Hz}), 0.68$ (d, $J=6.5 \mathrm{~Hz}), 1: 1.6,3 \mathrm{H}] .{ }^{13} \mathrm{C}\left\{{ }^{1} \mathrm{H}\right\} \operatorname{NMR}(126 \mathrm{MHz}, 1.6: 1$ mixture of rotamers $): \delta \quad 155.5,154.6,145.9$, $145.2,142.5,141.3,137.2,128.9,128.7,128.5,128.1,127.8,127.7,127.0,126.7,126.7,126.6,126.5$, $126.4,125.5,66.9,56.2,54.3,53.9,53.2,52.8,46.2,45.4,29.1,28.9,21.8,21.7,20.7,20.1,18.1$, 17.9.

For $(E)-3 \mathrm{~d}: \operatorname{TLC}\left(\mathrm{CH}_{2} \mathrm{Cl}_{2}\right.$-hexanes $\left.=1: 1\right): R_{f}=0.11 .{ }^{1} \mathrm{H}$ NMR $(500 \mathrm{MHz}, 1.8: 1$ mixture of rotamers): $\delta$ 7.12-7.34 $(\mathrm{m}, 15 \mathrm{H}),[5.24(\mathrm{~d}, J=12.0 \mathrm{~Hz}), 5.08(\mathrm{~d}, J=13.0 \mathrm{~Hz}), 1: 1.8,1 \mathrm{H}], 4.98-5.01$ $(\mathrm{m}, 2 \mathrm{H}),[4.66(\mathrm{dd}, J=12.0 \mathrm{~Hz}), 4.50(\mathrm{~d}, J=4.50 \mathrm{~Hz}), 1.8: 1,1 \mathrm{H}],[4.40(\mathrm{dt}, J=7.5,8.5 \mathrm{~Hz}), 4.29(\mathrm{dt}, J$ $=7.0,8.5 \mathrm{~Hz}), 1: 1.8,1 \mathrm{H}],[3.71(\mathrm{~d}, J=11.5 \mathrm{~Hz}), 3.68(\mathrm{~d}, J=11.5), 1: 1.8,1 \mathrm{H}], 2.74-2.78(\mathrm{~m}, 1 \mathrm{H})$, [2.54 (dd, $J=9.5 \mathrm{~Hz}), 2.31(\mathrm{dd}, J=9.5,12.5 \mathrm{~Hz}), 1: 1.8,1 \mathrm{H}],[2.13$ (septet, $J=7.0 \mathrm{~Hz}), 2.04$ (septet, $J$ $=7.5 \mathrm{~Hz}), 1: 1.8,1 \mathrm{H}],[1.59(\mathrm{~s}), 1.35(\mathrm{~s}), 1: 1.8,3 \mathrm{H}],[0.91(\mathrm{~d}, J=7.0 \mathrm{~Hz}), 0.84(\mathrm{~d}, J=7.0 \mathrm{~Hz}), 1: 1.8,3$ $\mathrm{H}],[0.91(\mathrm{~d}, J=7.0 \mathrm{~Hz}), 0.82(\mathrm{~d}, J=7.0 \mathrm{~Hz}), 1: 1.8,3 \mathrm{H}] .{ }^{13} \mathrm{C}\left\{{ }^{1} \mathrm{H}\right\}$ NMR $(126 \mathrm{MHz}, 1.8: 1$ mixture of rotamers): $\delta 155.6,154.6,145.9,145.3,145.2,142.9,142.0,137.4,137.1,128.8,128.7,128.7,128.6$, 
$128.5,128.2,128.1,127.0,126.7,126.7,126.6,126.5,124.3,123.7,67.0,66.8,56.1,55.2,54.6,53.2$ $52.8,45.8,45.0,36.6,21.6,21.4,21.3,21.3,14.3,14.0$.

\section{(E)-Benzyl 2-(2,3,3-trimethyl-1-butenyl)-4,4-diphenylpyrrolidine-1-carboxylate [(E)-3e].}

Colorless oil. TLC $\left(\mathrm{CH}_{2} \mathrm{Cl}_{2}\right.$-hexanes $\left.=3: 1\right): R_{f}=0.36 .{ }^{1} \mathrm{H}$ NMR $(500 \mathrm{MHz}, 2.3: 1$ mixture of rotamers, Figure S19): $\delta$ 7.16-7.38 (m, $15 \mathrm{H}),[5.29(\mathrm{~d}, J=12.5 \mathrm{~Hz}), 5.12(\mathrm{~d}, J=12.5 \mathrm{~Hz}), 1: 2.3], 5.03-5.08(\mathrm{~m}, 2$ H), [4.65 (d, $J=11.5 \mathrm{~Hz}), 4.48(\mathrm{~d}, J=9.5 \mathrm{~Hz}), 2.3: 1,1 \mathrm{H}],[4.47(\mathrm{dd}, J=8.0-15.0 \mathrm{~Hz}), 4.37(\mathrm{dd}, J=8.5$, 16.0 Hz), 1:2.3, $1 \mathrm{H}],[3.83(\mathrm{~d}, J=12.5 \mathrm{~Hz}), 3.81(\mathrm{~d}, J=11.5 \mathrm{~Hz}), 2.3: 1,1 \mathrm{H}], 2.82-2.88(\mathrm{~m}, 1 \mathrm{H}),[2.35$ (dd, $J=9.0,12.5 \mathrm{~Hz}), 2.30(\mathrm{dd}, J=9.0,12.0 \mathrm{~Hz}), 2.3: 1,1 \mathrm{H}],[1.65$ (s), 1.45 (s), 1:2.3, $1 \mathrm{H}]$, [0.97 (s), 0.89 (s), 1:2.3, $9 \mathrm{H}] .{ }^{13} \mathrm{C}\left\{{ }^{1} \mathrm{H}\right\}$ NMR (126 MHz, 2.3:1 mixture of rotamers, Figure S20): $\delta$ 155.6, 154.6, $145.9,145.4,145.3,144.5,143.6,137.4,137.1,128.8,128.8,128.7,128.6,128.5,128.2,128.1,127.9$, $127.8,127.0,126.7,126.7,126.6,126.5$. IR (neat, $\mathrm{cm}^{-1}$ ): 2963, 2869, 2696, 1411, 1352, 696. HRMS calcd (found) for $\mathrm{C}_{31} \mathrm{H}_{35} \mathrm{NO}_{2}\left(\mathrm{M}^{+}\right): 453.2668$ (453.2669).

Benzyl 2-(2-ethyl-1-octenyl)-4,4-diphenyl-pyrrolidine-1-carboxylate (3f). Colorless oil. Anal. calcd (found) for $\mathrm{C}_{34} \mathrm{H}_{41} \mathrm{NO}_{2}: \mathrm{H}, 8.34$ (8.27); C, 82.38 (82.52). IR (neat, $\mathrm{cm}^{-1}$ ): 2927, 2866, $1698,1411,1132,696$. NMR analysis was performed on an enantiomerically enriched $\geq 50: 1$ mixture of $(Z)-\mathbf{3 f} /(E)-\mathbf{3 f}$ and on a racemic $\geq 50: 1$ mixture of $(E)-\mathbf{3 f} /(Z)-\mathbf{3 f}$.

For (Z)-3f: $\operatorname{TLC}\left(\mathrm{CH}_{2} \mathrm{Cl}_{2}\right): R_{f}=0.65 .{ }^{1} \mathrm{H}$ NMR $(500 \mathrm{MHz}, 1.8: 1$ mixture of rotamers $): \delta$ 7.16$7.36(\mathrm{~m}, 15 \mathrm{H}),[5.26(\mathrm{~d}, J=12.0 \mathrm{~Hz}), 5.13(\mathrm{~d}, J=12.0 \mathrm{~Hz}), 1: 1.8,1 \mathrm{H}], 4.99-5.03(\mathrm{~m}, 2 \mathrm{H}),[4.70(\mathrm{dt}, J$ $=1.0,11.5 \mathrm{~Hz}), 4.56(\mathrm{~d}, J=11.5 \mathrm{~Hz}), 1: 1.8,1 \mathrm{H}],[3.74(\mathrm{~d}, J=12.5 \mathrm{~Hz}), 3.72(\mathrm{~d}, J=11.5 \mathrm{~Hz}), 1: 1.8,1$ $\mathrm{H}], 2.76-2.83(\mathrm{~m}, 1 \mathrm{H}),[2.37(\mathrm{dd}, J=9.5,12.0 \mathrm{~Hz}), 2.32(\mathrm{dd}, J=9.5,12.0 \mathrm{~Hz}), 1.8: 1,1 \mathrm{H}], 2.22-2.26$ (m, 0.4 H), 1.84-2.02 (m, 2.6 H), [1.61-1.64 (m), 1.40-1.46 (m), 1.8:1, $1 \mathrm{H}], 1.11-1.28(\mathrm{~m}, 9 \mathrm{H}),[0.94(\mathrm{t}$, $J=7.5 \mathrm{~Hz}), 0.88(\mathrm{t}, J=7.5 \mathrm{~Hz}), 1: 1.8,3 \mathrm{H}], 0.82-0.88(\mathrm{~m}, 3 \mathrm{H}) .{ }^{13} \mathrm{C}\left\{{ }^{1} \mathrm{H}\right\} \mathrm{NMR}(126 \mathrm{MHz}, 1.8: 1$ mixture of rotamers): $\delta 155.6,154.6,145.9,145.2,145.1,143.5,142.5,137.3,137.1,128.8,128.7,128.4$, $128.2,127.8,127.0,126.7,126.6,126.6,126.5,125.2,124.4,66.9,66.8,56.2,55.0,54.5,53.2,52.7$, $46.2,45.5,31.9,31.8,31.0,30.7,29.6,29.5,29.4,29.0,28.6,22.8,22.8,14.3,12.6,12.5$. 
For (E)-3f: $\operatorname{TLC}\left(\mathrm{CH}_{2} \mathrm{Cl}_{2}\right): R_{f}=0.67 .{ }^{1} \mathrm{H}$ NMR $(500 \mathrm{MHz}, 1.7: 1$ mixture of rotamers): $\delta \quad 7.15-$ $7.36(\mathrm{~m}, 15 \mathrm{H}),[5.26(\mathrm{~d}, J=12.5 \mathrm{~Hz}), 5.10(\mathrm{~d}, J=12.5 \mathrm{~Hz}), 1: 1.7,1 \mathrm{H}], 5.04(\mathrm{~d}, J=12.5 \mathrm{~Hz}, 0.4 \mathrm{H})$, 4.96-5.00 (m, 1.6 H), [4.70 (dd, $J=1.0,11.0 \mathrm{~Hz}), 4.55(\mathrm{~d}, J=11.0 \mathrm{~Hz}), 1.7: 1, \mathrm{H}],[4.45(\mathrm{dt}, J=7.5,8.5$ $\mathrm{Hz}), 4.33(\mathrm{~J}=7.0,9.0 \mathrm{~Hz}), 1: 1.7,1 \mathrm{H}],[3.72(\mathrm{~d}, J=12.0 \mathrm{~Hz}), 3.69(\mathrm{~d}, J=12.0 \mathrm{~Hz}), 1: 1.7,1 \mathrm{H}], 4.74-$ $4.82(\mathrm{~m}, 1 \mathrm{H}),[2.36(\mathrm{dd}, J=10.0,12.5 \mathrm{~Hz}), 2.30(\mathrm{dd}, J=9.5,12.5 \mathrm{~Hz}), 1: 1.7,1 \mathrm{H}], 2.12-2.19(\mathrm{~m}, 0.4$ H), 1.83-2.03 (m, $3 \mathrm{H}), 1.68-1.75(\mathrm{~m}, 0.6 \mathrm{H}), 1.18-1.33(\mathrm{~m}, 8 \mathrm{H}), 0.96(\mathrm{t}, J=7.5 \mathrm{~Hz}, 0.4 \mathrm{H}), 0.85-0.88$ $(\mathrm{m}, 3 \mathrm{H}), 0.78(\mathrm{t}, J=7.5 \mathrm{~Hz}, 3 \mathrm{H}) .{ }^{13} \mathrm{C}\left\{{ }^{1} \mathrm{H}\right\} \mathrm{NMR}(126 \mathrm{MHz}, 1.7: 1$ mixture of rotamers): $\delta 155.5,154.6$, $145.9,145.3,145.2,143.4,142.3,137.3,137.1,128.8,128.7,128.4,128.2,127.8,127.0,126.7,126.7$ 126.6, 126.5, 125.8, 125.0, 66.9, 66.8, 56.1, 54.9, 54.4, 53.2, 52.8, 46.2, 45.5, 36.4, 36.3, 32.0, 29.3, $28.1,28.0,23.8,23.5,22.8,14.3,13.7,13.5$.

Benzyl 2-(2-methyl-1-octenyl)-pyrrolidine-1-carboxylate (3g). Colorless oil. Anal. calcd (found) for $\mathrm{C}_{21} \mathrm{H}_{31} \mathrm{NO}_{2}: \mathrm{H}, 9.48$ (9.53); C, 76.55 (76.46). IR (neat, $\mathrm{cm}^{-1}$ ): 2925, 2862, 1700, 1410, 1351, 1103. NMR analysis was performed on an enantiomerically enriched $6.1: 1$ mixture of $(Z)-3 \mathbf{g} /(E)-$ $\mathbf{3 g}$ and on a racemic $5.7: 1$ mixture of $(E)-\mathbf{3 g} /(Z)-\mathbf{3 g}$.

For (Z)-3g: $\operatorname{TLC}\left(\mathrm{CH}_{2} \mathrm{Cl}_{2}\right.$-hexanes $\left.=3: 1\right): R_{f}=0.14 .{ }^{1} \mathrm{H}$ NMR $(500 \mathrm{MHz}, 1.6: 1$ mixture of rotamers): $\delta$ 7.21-7.36 (m, $5 \mathrm{H}), 5.13(\mathrm{~d}, J=12.5 \mathrm{~Hz}, 0.6 \mathrm{H}), 4.99-5.12(\mathrm{~m}, 2.4 \mathrm{H})$, [4.56 (br s), 4.47 (br s), 1:1.6, $1 \mathrm{H}], 3.40-3.48(\mathrm{~m}, 2 \mathrm{H}), 2.38(\mathrm{br} \mathrm{s}, 0.4 \mathrm{H}), 1.17-2.10(\mathrm{~m}, 16.6 \mathrm{H}), 0.84-0.88(\mathrm{~m}, 3 \mathrm{H}) .{ }^{13} \mathrm{C}\left\{{ }^{1} \mathrm{H}\right\}$ NMR (126 MHz, 1.5:1 mixture of rotamers): $\delta 137.4,136.5,128.5,128.1,127.9,126.9,126.2,66.8$, $55.6,55.2,46.9,46.5,39.8,34.0,32.1,32.0,29.6,28.2,22.9,14.3$.

For (E)-3g: $\operatorname{TLC}\left(\mathrm{CH}_{2} \mathrm{Cl}_{2}\right.$-hexanes $\left.=3: 1\right): R_{f}=0.14 .{ }^{1} \mathrm{H}$ NMR $(500 \mathrm{MHz}, 1.5: 1$ mixture of rotamers): $\delta$ 7.26-7.35 (m, $5 \mathrm{H})$, 5.02-5.15 (m, $3 \mathrm{H}),[4.56(\mathrm{br} \mathrm{s}, 4.48(\mathrm{br} \mathrm{s}), 1: 1.5,1 \mathrm{H}], 3.41-3.46(\mathrm{~m}, 2$ $\mathrm{H}), 1.20-2.08(\mathrm{~m}, 17 \mathrm{H}), 0.86(\mathrm{t}, J=7.0 \mathrm{~Hz}, 3 \mathrm{H}) .{ }^{13} \mathrm{C}\left\{{ }^{1} \mathrm{H}\right\} \mathrm{NMR}(126 \mathrm{MHz}, 1.5: 1$ mixture of rotamers $): \delta$ $155.4,137.4,136.2,128.5,128.1,127.9,126.0,125.5,66.8,55.8,55.4,46.9,46.5,39.8,31.9,29.2$, $27.9,22.9,14.3$ 


\section{Determination of Absolute Configuration of $(R, E)-3 a$ and $(R, Z)-3 a$}

The absolute configuration of the major diastereomers formed in the hydroamination of $\mathbf{2 a}$ catalyzed by $(S)-1 / \mathrm{AgClO}_{4}$ were assigned as $(R, Z)-3 \mathbf{a}$ and $(R, E)-3 \mathbf{a}$ on the basis of the experiments described below and outlined in Schemes S2 and S3. We first established the homochiral nature of $(R, E)-3 \mathbf{a}$ and $(R, Z)-3 \mathbf{a}$ by converting a $3.1: 1$ mixture of $(R, Z)-3 \mathbf{a}(96 \%$ ee) and $(R, E)-3 \mathbf{a}(76 \%$ ee) to $(R)$ benzyl 2-formyl-4,4-diphenylpyrrolidine-1-carboxylate [(R)-S11, 85\% ee, Figure S21) via ozonolysis followed by reductive work up (Scheme S2). The enantiopurity of $(R)-\mathbf{S 1 1} 85 \%$ ee is only slightly lower than the value of $90 \%$ ee calculated for conversion of a homochiral 3.1:1 mixture of $(Z)-3 \mathbf{a}$ and $(E)-3 \mathbf{a}$ to $\mathbf{S 1 1}$ but differs significantly from the value of $52 \%$ ee calculated for conversion of a $3.1: 1$ mixture of either $(R, Z)-\mathbf{3} \mathbf{a}$ and $(S, E)-\mathbf{3 a}$ or $(S, Z)-\mathbf{3} \mathbf{a}$ and $(R, E)-3 \mathbf{a}$ to $\mathbf{S 1 1 .}$

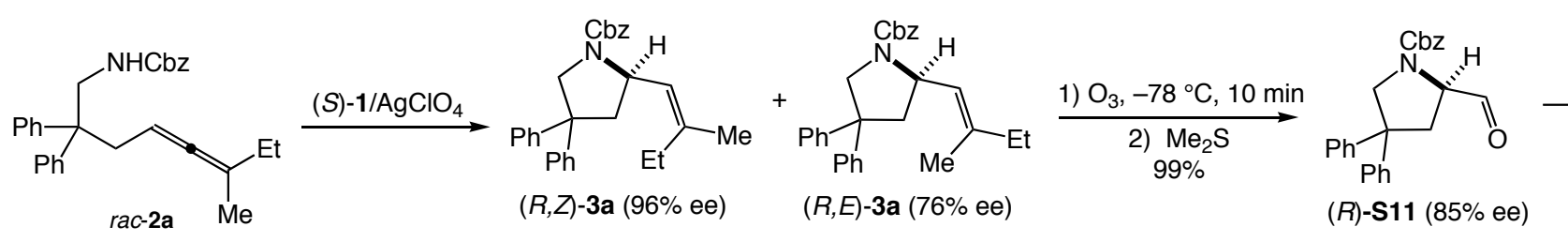

$\mathrm{Z} / \mathrm{E}=3.1: 1$

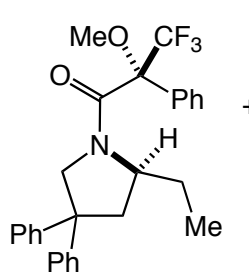

$(R, S)-\mathbf{S} 13$

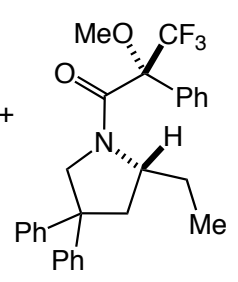

$(R, R)-\mathbf{S} 13$

\section{Scheme S2}

$11.1: 1$
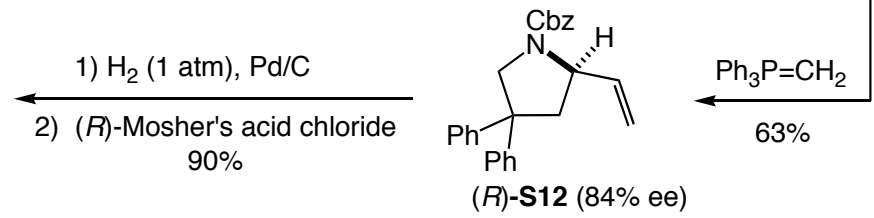

(R)-S12 (84\% ee)

We then established that the absolute configuration of $(R, Z)-3 a$ and $(R, E)-3 \mathbf{a}$ formed via reaction of $2 \mathrm{a}$ with $(S)-1 / \mathrm{AgClO}_{4}$ was $\mathrm{R}$. In one set of experiments, rac-S12 ${ }^{2}$ was hydrogenated to cleave the $\mathrm{Cbz}$ group and reduce the vinyl group [note that hydrogenation of the exocyclic vinyl group inverts the CIP priorities of the exocyclic group and the $C(3)$ pyrrolidine carbon atom] and then treated with $(R)$-Mosher's acid chloride to form a 1:1 mixture of $(R, R)-\mathbf{S} 13$ and $(R, S)$-S13 (Scheme S3). Slow 
evaporation of an ether solution of a 1:1 mixture of $(R, R)-\mathbf{S} 13$ and $(R, S)$-S13 gave a large, colorless crystal of $(R, S)$-S13 suitable for X-ray diffraction. The crystal was cut and one fragment was subjected to single crystal X-ray analysis, which established the configuration as $(R, S)$-S13 (Figure S22) and the second fragment was analyzed by HPLC (Figure S23, middle trace). In a separate set of experiments, (R)-S11 [85\% ee, obtained via ozonolysis of a 3.1:1 mixture of $(R, Z)-3 \mathbf{a}(96 \%$ ee) and $(R, E)-3 \mathbf{a}(76 \%$ ee)] was converted to (R)-S12 (84\% ee, Figure S24, right trace) via Wittig olefination (Scheme S2). Reduction of $(R)-\mathrm{S} 12\left(\mathrm{H}_{2}, \mathrm{Pd} / \mathrm{C}\right)$ [note that hydrogenation of the exocyclic vinyl group inverts the CIP priorities of the exocyclic group and the $\mathrm{C}(3)$ pyrrolidine carbon atom] followed by treatment with $(R)$ Mosher's acid chloride gave a 11.1:1 mixture of $(R, S)-\mathbf{S 1 3}$ and $(R, R)-\mathbf{S 1 3}$ as determined by HPLC analysis (Figure S23, right trace). Comparison of the HPLC traces of the 11.1:1 mixture of $(R, S)$-S13 and $(R, R)$-S13 with the HPLC trace of pure $(R, S)$-S13 established the configuration of the $\mathrm{C}(2)$ stereocenter of the predominant diasteromer as $\mathbf{S}$. Therefore, we conclude that reaction of $2 \mathbf{a}$ with $(S)$ $1 / \mathrm{AgClO}_{4}$ forms a 3.1:1 mixture of $(R, Z)-3 \mathbf{a}$ and $(R, E)-3 \mathbf{a}$.

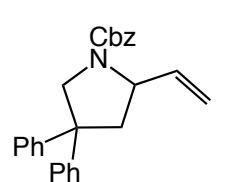

(土)-S12 $\underset{\text { 2) }}{\stackrel{\text { 1) } \mathrm{H}_{2}(1 \mathrm{~atm}), \mathrm{Pd} / \mathrm{C}}{\longrightarrow} \text {-Mosher's acid chloride }}$
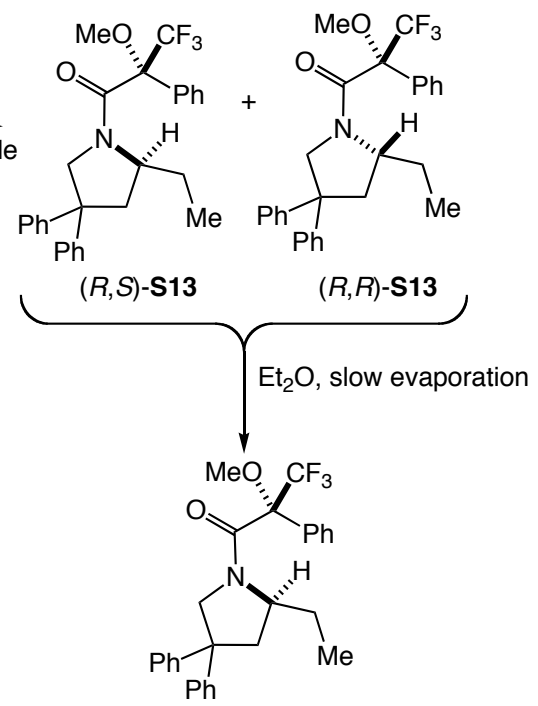

$(R, S)-\mathrm{S} 13$

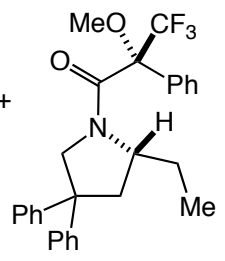

$(R, R)-\mathrm{S} 13$

\section{Scheme S3}

(


then sparged with nitrogen for $3 \mathrm{~min}$, treated with $\mathrm{Me}_{2} \mathrm{~S}(209 \mathrm{mg}, 3.36 \mathrm{mmol})$, warmed to room temperature, stirred for $1 \mathrm{~h}$, and concentrated under reduced pressure. The resulting oily residue was chromatographed $\left(\mathrm{Et}_{2} \mathrm{O}-\mathrm{CH}_{2} \mathrm{Cl}_{2}=0: 100 \rightarrow 1: 1\right)$ to give $(R)-\mathrm{S} 11$ as a colorless oil $(86.0 \mathrm{mg}, 99 \%)$. The enatiomeric purity of $(R)-\mathrm{S} 11$ was determined by HPLC analysis (Figure S21). TLC $\left(\mathrm{CH}_{2} \mathrm{Cl}_{2}\right): R_{f}=$ 0.33. ${ }^{1} \mathrm{H}$ NMR (400 MHz, 1:1 mixture of rotamers, Figure S25): $\delta 9.43(\mathrm{~d}, J=2.4 \mathrm{~Hz}, 0.5 \mathrm{H}), 9.32(\mathrm{~d}, J$ $=2.8 \mathrm{~Hz}, 0.5 \mathrm{H}), 7.15-7.45(\mathrm{~m}, 15 \mathrm{H}), 5.30(\mathrm{~d}, J=12.4 \mathrm{~Hz}, 0.5 \mathrm{H}), 5.21(\mathrm{~d}, J=12.4 \mathrm{~Hz}, 0.5 \mathrm{H}), 5.17(\mathrm{~s}$, $1 \mathrm{H}), 4.44(\mathrm{~d}, J=11.6 \mathrm{~Hz}, 0.5 \mathrm{H}), 4.30(\mathrm{~d}, J=11.6 \mathrm{~Hz}, 0.5 \mathrm{H}), 4.17(\mathrm{dt}, J=2.0,7.6 \mathrm{~Hz}, 0.5 \mathrm{H}), 4.08(\mathrm{dt}$, $J=2.8,8.0 \mathrm{~Hz}, 0.5 \mathrm{H}), 4.12(\mathrm{~d}, J=2.8 \mathrm{~Hz}, 0.5 \mathrm{H}), 4.10(\mathrm{~d}, J=2.4 \mathrm{~Hz}, 0.5 \mathrm{H}), 2.67-2.88(\mathrm{~m}, 2 \mathrm{H})$. ${ }^{13} \mathrm{C}\left\{{ }^{1} \mathrm{H}\right\}$ NMR $(101 \mathrm{MHz}, 1: 1$ mixture of rotamers, Figure S26): $\delta \quad 200.5,200.4,155.4,154.6,144.07$, $144.04,144.01,143.92,136.5,136.1,128.94,128.93,128.89,128.88,128.80,128.70,128.48,128.32$ 127.97, 127.24, 127.03, 126.83, 126.82, 126.48, 67.8, 67.7, 64.3, 64.0, 56.3, 56.1, 53.6, 52.9, 41.0, 40.0. IR (neat, $\mathrm{cm}^{-1}$ ): $3729,3363,1690,1420,1355,1136,694$. HRMS calcd (found) for $\mathrm{C}_{25} \mathrm{H}_{24} \mathrm{NO}_{3}$ $\left(\mathrm{MH}^{+}\right): 386.1756(386.1755)$.

$(\boldsymbol{R}, \boldsymbol{S})$-S13 and $(\boldsymbol{R}, \boldsymbol{R})$-S13. $n$-BuLi $(2.5 \mathrm{M}$ in hexanes, $0.13 \mathrm{~mL}, 0.32 \mathrm{mmol})$ was added dropwise to a suspension of methyltriphenylphosphonium bromide $(112.5 \mathrm{mg}, 0.32 \mathrm{mmol})$ in THF $(6 \mathrm{~mL})$ at -78 ${ }^{\circ} \mathrm{C}$. The resulting mixture was warmed to room temperature and stirred for $1 \mathrm{~h}$. The reaction was cooled down to $-78{ }^{\circ} \mathrm{C}$, and treated with the solution of $(R)-\mathrm{S} 11(79.5 \mathrm{mg}, 0.21 \mathrm{mmol}, 85 \%$ ee) in THF $(4 \mathrm{~mL})$. The resulting yellow suspension was warmed to room temperature overnight with stirring, diluted with $\mathrm{Et}_{2} \mathrm{O}(20 \mathrm{~mL})$, quenched with saturated $\mathrm{NH}_{4} \mathrm{Cl}(20 \mathrm{~mL})$, and extracted with $\mathrm{Et}_{2} \mathrm{O}(3 \times 40 \mathrm{~mL})$. The combined organic fractions were washed with brine $(50 \mathrm{~mL})$, dried $\left(\mathrm{MgSO}_{4}\right)$, and concentrated. The residue was chromatographed (EtOAc-hexanes $=20: 1 \rightarrow 5: 1)$ to give $(R)-\mathbf{S 1 2}{ }^{2}(50.4 \mathrm{mg}, 63 \%)$ as a colorless oil. The enantiomeric purity of $(R)$-S12 was determined by HPLC equipped with a chrial stationary phase (84\% ee, Figure S24, right trace).

A mixture of $(R)-\mathrm{S} 12(42.1 \mathrm{mg}, 0.11 \mathrm{mmol}, 84 \% \mathrm{ee}), \mathrm{Pd} / \mathrm{C}(20 \mathrm{mg})$, and $\mathrm{MeOH}(2 \mathrm{~mL})$ was stirred under $\mathrm{H}_{2}(1 \mathrm{~atm})$ at room temperature for $6 \mathrm{~h}$, filtered through a pad of Celite $(\sim 5 \mathrm{~cm})$ in a pipet, 
washed with ether ( 20 mL), and concentrated to provide crude (S)-2-ethyl-4,4-diphenylpyrrolidine [(S)S14] as a colorless oil that was used in the subsequent step without further purification.

$(R)-(-)-\alpha-$ Methoxy- $\alpha$-(trifluoromethyl)phenylacetyl chloride $(33.4 \mathrm{mg}, 0.132 \mathrm{mmol})$ was added slowly to a solution of (S)-S14 (all material obtained in previous step), DMAP (1.3 mg, $0.01 \mathrm{mmol}$ ), and $\mathrm{Et}_{3} \mathrm{~N}(16.7 \mathrm{mg}, 0.17 \mathrm{mmol})$ in $\mathrm{CH}_{2} \mathrm{Cl}_{2}(2.2 \mathrm{~mL})$ at $0{ }^{\circ} \mathrm{C}$. The resulting mixture was warmed to room temperature overnight with stirring, diluted with ether $(15 \mathrm{~mL})$, quenched with saturated $\mathrm{NH}_{4} \mathrm{Cl}(15 \mathrm{~mL})$, and extracted with $\mathrm{Et}_{2} \mathrm{O}(3 \times 30 \mathrm{~mL})$. The combined organic fractions were dried $\left(\mathrm{MgSO}_{4}\right)$, and concentrated. The residue was chromatographed $\left(\mathrm{CH}_{2} \mathrm{Cl}_{2}\right.$-hexanes $\left.=1: 1 \rightarrow 5: 1\right)$ to give a 11.1:1 mixture of $(R, S)$-S13 and $(R, R)-\mathrm{S} 13(46.2 \mathrm{mg}, 90 \%)$ as a white solid. $\mathrm{mp}=204{ }^{\circ} \mathrm{C} . \quad \mathrm{TLC}\left(\mathrm{CH}_{2} \mathrm{Cl}_{2^{-}}\right.$ hexanes $=1: 1): \quad R_{f}=0.23 . \quad$ IR (neat, $\left.\mathrm{cm}^{-1}\right): 3625,2981,2872,1648,1418,1146$. HRMS calcd (found) for $\mathrm{C}_{28} \mathrm{H}_{28} \mathrm{~F}_{3} \mathrm{NO}_{2}\left(\mathrm{M}^{+}\right)$: 467.2072 (467.2073). NMR assignments for $(R, S)$-S13 and $(R, R)-\mathrm{S} 13$ were made from analysis of this mixture.

For $(\boldsymbol{R}, \boldsymbol{S})-\mathrm{S} 13:{ }^{1} \mathrm{H}$ NMR (Figure S27): $\delta$ 6.47-7.49 (m, $\left.15 \mathrm{H}\right), 4.19(\mathrm{dd}, J=2.0,11.6 \mathrm{~Hz}, 1 \mathrm{H})$, 4.05-4.12 (m, $1 \mathrm{H}), 3.71(\mathrm{~d}, J=1.6 \mathrm{~Hz}, 3 \mathrm{H}), 3.68(\mathrm{~d}, J=11.6 \mathrm{~Hz}, 1 \mathrm{H}), 2.96(\mathrm{ddd}, J=1.6,6.8,12.8 \mathrm{~Hz}$, $1 \mathrm{H}), 2.33-2.43(\mathrm{~m}, 1 \mathrm{H}), 2.07(\mathrm{dd}, J=10.6,13.0 \mathrm{~Hz}, 1 \mathrm{H}), 1.41-1.52(\mathrm{~m}, 1 \mathrm{H}), 0.96(\mathrm{t}, J=7.4 \mathrm{~Hz}, 3 \mathrm{H})$. ${ }^{13} \mathrm{C}\left\{{ }^{1} \mathrm{H}\right\}$ NMR (Figure S28): $\delta \quad 164.5,146.3,143.8,132.7,129.4,128.7,128.6,127.1,127.0,126.7$, $126.6,126.2,126.1,125.2,122.3,84.7,84.5,59.9,57.1,55.0,53.2,41.6,26.3,10.0$.

For $(\boldsymbol{R}, \boldsymbol{R})-\mathrm{S} 13:{ }^{1} \mathrm{H}$ NMR (Figure S27): $\delta \quad 6.75-7.54(\mathrm{~m}, 15 \mathrm{H}), 4.37(\mathrm{dq}, J=3.0,8.4 \mathrm{~Hz}, 1 \mathrm{H})$, $4.06(\mathrm{dd}, J=1.6,11.2 \mathrm{~Hz}, 1 \mathrm{H}), 3.05-3.14(\mathrm{~m}, 1 \mathrm{H}), 3.01(\mathrm{~d}, J=11.2 \mathrm{~Hz}, 1 \mathrm{H}), 2.80(\mathrm{~d}, J=1.2 \mathrm{~Hz}, 3 \mathrm{H})$, 2.29-2.43 (m, $1 \mathrm{H}), 1.88(\mathrm{dd}, J=8.0,13.2 \mathrm{~Hz}, 1 \mathrm{H}), 1.17-1.25(\mathrm{~m}, 1 \mathrm{H}), 0.90(\mathrm{t}, J=7.4 \mathrm{~Hz}, 3 \mathrm{H})$. ${ }^{13} \mathrm{C}\left\{{ }^{1} \mathrm{H}\right\}$ NMR (Figure S28): $\delta \quad 164.3,146.5,145.8,134.5,128.7,128.6,128.4,127.3,127.2,126.9$, 126.6, 126.5, 125.2, 122.3, 84.8, 84.5, 60.2, 57.5, 55.0, 53.9, 41.0, 25.6, 9.9.

\section{Control and Comparison Experiments}


(1) Phosphine ligand alone. Treatment of $(R)-2 a(44 \%$ ee, $0.075 \mathrm{M})$ with $(S)-1(5 \mathrm{~mol} \%)$ in $m$-xylene at $23{ }^{\circ} \mathrm{C}$ for $24 \mathrm{~h}$ led to no consumption of $(R)-2 \mathrm{a}$. Analysis of the filtered and concentrated reaction mixture using HPLC equipped with chrial support revealed complete retention of configuration (44\% ee).

(2) Silver alone. Treatment of $(R)-2 \mathrm{a}(44 \% \mathrm{ee}, 0.075 \mathrm{M})$ with $\mathrm{AgClO}_{4}(15 \mathrm{~mol} \%)$ in $m$-xylene at $23{ }^{\circ} \mathrm{C}$ for $24 \mathrm{~h}$ led to no consumption of $(R)-2 \mathrm{a}$ as determined by $\mathrm{GC}$ analysis. Analysis of the filtered and concentrated reaction mixture using HPLC equipped with chrial support revealed complete retention of configuration ( $44 \%$ ee).

(3) Silver + phosphine ligand. Treatment of $(R)-2 \mathbf{a}(44 \%$ ee, $0.075 \mathrm{M})$ with $\mathrm{AgClO}_{4}(15 \mathrm{~mol}$ $\%)$ and (S)-3,5-t-Bu-4-MeO-MeOBIPHEP (7.5 mol\%) in $m$-xylene at $23{ }^{\circ} \mathrm{C}$ for $24 \mathrm{~h}$ led to no consumption of $(R)-2 a$. Analysis of the filtered and concentrated reaction mixture using HPLC equipped with chrial support revealed complete retention of configuration $(44 \%$ ee). Similarly, treatment of $(R)-2 a(44 \%$ ee, $0.075 \mathrm{M})$ with AgOTf (10 mol \%) and (S)-3,5-t-Bu-4-MeO-MeOBIPHEP (5 mol \%) in $m$-xylene at $23{ }^{\circ} \mathrm{C}$ for $24 \mathrm{~h}$ led to neither consumption nor racemization of $(R)-2 \mathrm{a}$.

(4) Acid + phosphine ligand. Treatment of $(R)-2 a(44 \%$ ee, $0.075 \mathrm{M})$ with $(S)-3,5-t-B u-4-$ MeO-MeOBIPHEP (5 mol\%) and $\mathrm{HClO}_{4}(70 \%, 10 \mathrm{~mol} \%)$ in $m$-xylene at $23{ }^{\circ} \mathrm{C}$ for $9 \mathrm{~h}$ led to $35 \%$ consumption of $(R)-2 \mathbf{a}$. Analysis of the filtered and concentrated reaction mixture using HPLC equipped with chrial support revealed complete retention of configuration ( $44 \%$ ee) and formation of a racemic 2:1 mixture of $(E)-3 \mathbf{a}$ and $(Z)-3 a$. Similarly, treatment of $(R)-2 a(44 \%$ ee, $0.075 \mathrm{M})$ with $(S)-3,5-$ $t$-Bu-4-MeO-MeOBIPHEP (5 mol \%) and TfOH $(70 \%, 10 \mathrm{~mol} \%)$ in $m$-xylene at $23{ }^{\circ} \mathrm{C}$ for $24 \mathrm{~h}$ led to $58 \%$ consumption of $(R)-2 a$. Analysis of the filtered and concentrated reaction mixture using HPLC equipped with chrial support revealed nominal racemization ( $26 \%$ ee) and formation of a racemic $1.5: 1$ mixture of $(E)-3 \mathbf{a}$ and (Z)-3a. Therefore, acid-catalyzed processes can be ruled out as a source of enantiomerically enriched (Z)-3a and $(E)-\mathbf{3} \mathbf{a}$ and as a pathway for the racemization of $\mathbf{2} \mathbf{a}$.

(5) Cationic platinum complex. Treatment of $(R)-2 a(44 \%$ ee, $0.075 \mathrm{M})$ with a catalytic $1: 1$ mixture of $\mathrm{Pt}[(S)-3,5-t-\mathrm{Bu}-4-\mathrm{MeO}-\mathrm{MeOBIPHEP}] \mathrm{Cl}_{2}{ }^{10}(10 \mathrm{~mol} \%)$ and AgOTf $(10 \mathrm{~mol} \%)$ in $m$-xylene at 
$23{ }^{\circ} \mathrm{C}$ for $24 \mathrm{~h}$ led to no consumption of $(R)-2 \mathrm{a}$. Analysis of the filtered and concentrated reaction mixture using HPLC equipped with chrial support revealed complete retention of configuration (44\% ee).

(6) Stability of vinyl pyrroldines under reaction conditions. A suspension of (S)-1 (12.1 $\left.\mathrm{mg}, 7.5 \times 10^{-3} \mathrm{mmol}\right), \mathrm{AgClO}_{4}\left(3.1 \mathrm{mg}, 1.5 \times 10^{-2} \mathrm{mmol}\right)$, and a 3.1:1 mixture of $(R, Z)-3 \mathbf{a}$ and $(R, E)-3 \mathbf{a}$ in $m$-xylene $(1.0 \mathrm{~mL})$ was stirred at $23{ }^{\circ} \mathrm{C}$ for two weeks. HPLC analysis of the resulting mixture revealed a 3.1:1 mixture of $(R, Z)-3 \mathbf{a}$ and $(R, E)-3 \mathbf{a}$.

\section{Concentration versus time plots}

A mixture of $(S)-1\left(12.1 \mathrm{mg}, 7.5 \times 10^{-3} \mathrm{mmol}\right)$ and $\mathrm{AgClO}_{4}\left(3.1 \mathrm{mg}, 15 \times 10^{-3} \mathrm{mmol}\right)$ in $\mathrm{m}$-xylene $(0.6 \mathrm{~mL})$ was stirred at room temperature for $5 \mathrm{~min}$, treated with a solution of $2 \mathrm{a}(127.7 \mathrm{mg}, 0.30 \mathrm{mmol})$ in $m$-xylene $(1.4 \mathrm{~mL})$, and the resulting suspension was stirred at $23^{\circ} \mathrm{C}$. Aliquots $(25 \mu \mathrm{L})$ were removed at regular intervals and analyzed by HPLC equipped with chiral stationary phase. Aliquots were immediately loaded onto a short plug $(\sim 2 \mathrm{~cm})$ of silica gel in a pipet and eluted with ether $(15 \mathrm{~mL})$, concentrated, and dried under high vacuum. The resulting residue was dissolved in dry hexanes $(0.5$ $\mathrm{mL}$ ) for HPLC analysis, a representative HPLC appears in Figure S29.

\section{X-ray crystal structure of $(R, S)-S 13$.}

Colorless crystals of $(R, S)-S 13$ were obtained by slow evaporation of a ether solution of a 1:1 mixture of $(R, S)$-S13 and $(R, R)$-S13 at room temperature. Diffraction data were obtained with graphite monochromated Mo K $\alpha$ radiation $(I=0.71073 \AA)$ on a Bruker Kappa Apex II diffractometer using the $\omega$ scan mode (Figure S22). Of the 3241 reflections, 3008 independent, observed reflections $(I>2.5 \sigma(I))$ were obtained with maximum $h, k, I$ values of 17,17 , and 17 , respectively. An absorption correction was applied (semi-empirical from equivalents). The solution method was SHELXS-97 (Sheldrick, 1990) and the refinement method was SHELXL-97 (Sheldrick, 1997). Tables of crystal data and structure 
refinement (Table S1), atomic coordinates and equivalent isotropic displacement parameters (Table S2), bond lengths and angles (Table S3), anisotropic displacement parameters (Table S4), hydrogen coordinates and isotropic displacement parameters (Table S5), and torsion angles (Table S6) are provided.

Figure S22. ORTEP diagram of the X-ray crystal structure of $(R, S)-S 13$.

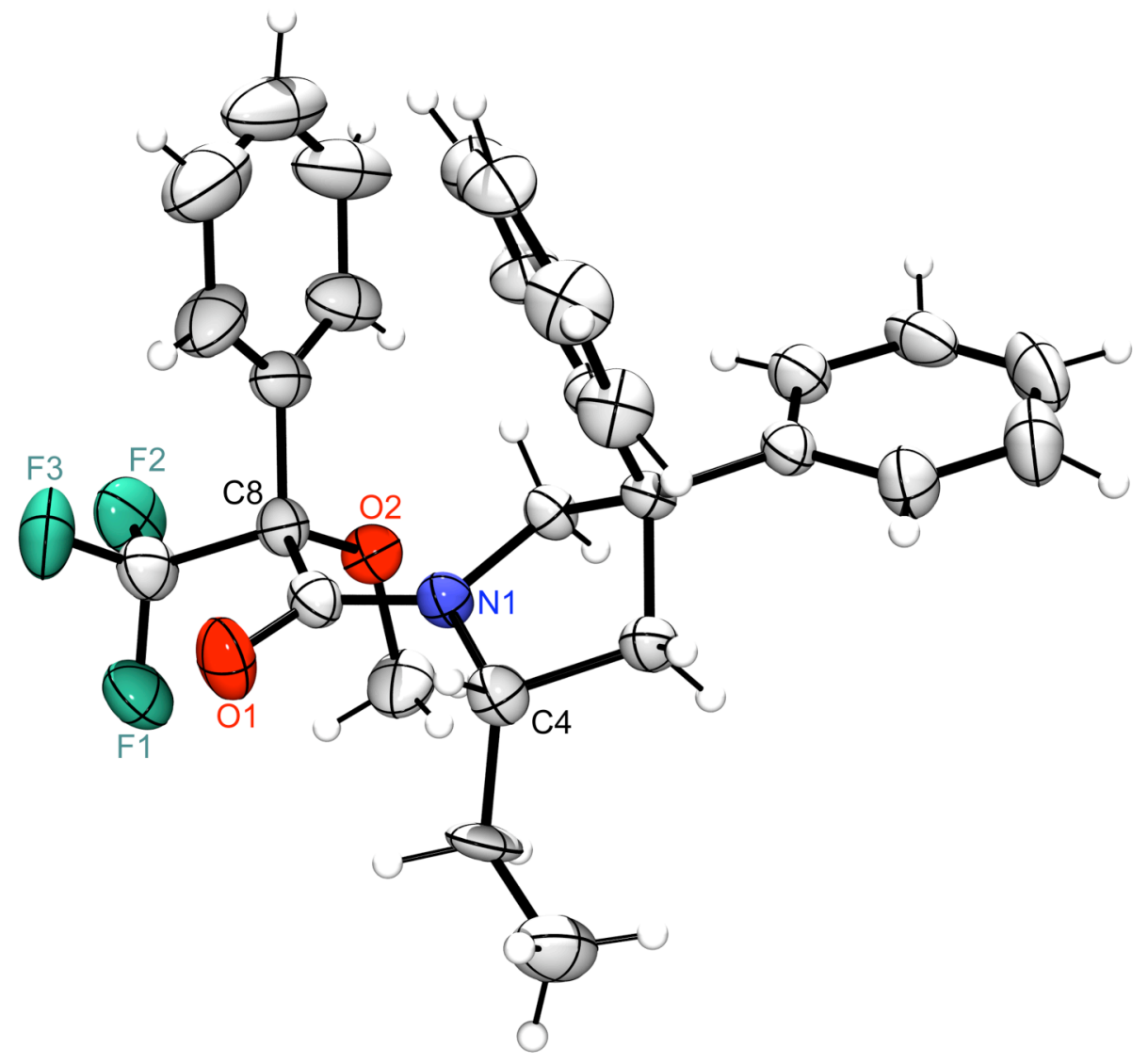


Table S1. Crystal data and structure refinement for $(R, S)-S 13$.

\begin{tabular}{ll}
\hline empirical formula & $\mathrm{C}_{28} \mathrm{H}_{28} \mathrm{~F}_{3} \mathrm{NO}_{2}$ \\
fw & 467.51 \\
temp $(\mathrm{K})$ & $298(2)$ \\
crystal size $(\mathrm{mm})$ & $0.27 \times 0.20 \times 0.14$ \\
color and habit & colorless prism \\
wavelength $(\AA)$ & 0.71073 \\
crystal system & orthorhombic \\
space group & $\mathrm{P} 22_{1} 2_{1} 2_{1}$ \\
$a(\AA)$ & $13.062(7)$ \\
$b(\AA)$ & $13.610(7)$ \\
$c(\AA)$ & $13.627(7)$ \\
$V\left(\AA^{3}\right)$ & $2422(2)$ \\
$Z$ & 4 \\
$D_{\mathrm{c}}\left(\mathrm{mg} / \mathrm{m}^{3}\right)$ & 1.282 \\
abs. coeff. (mm $\left.{ }^{-1}\right)$ & 0.096 \\
$F(000)$ & 984 \\
$2 \theta$ limits $($ deg $)$ & $2.11 \leq 2 \theta \leq 27.87^{\circ}$. \\
Completeness to theta $=27.87^{\circ}$ & $99.9 \%$ \\
Max. and min. trans. & 0.9867 and 0.9747 \\
no. param. & 308 \\
GOF & 1.075 \\
$R(\mathrm{~F})$ & 0.0348, \\
$R_{w}(\mathrm{~F})$ & 0.0920 \\
abs. structure param. & $-1.3(7)$ \\
largest res. density $\left(\mathrm{e} / \AA^{3}\right)$ & 0.214 and -0.196 \\
& \\
\hline
\end{tabular}


Table S2. Atomic coordinates $\left(\times 10^{4}\right)$ and equivalent isotropic displacement parameters $\left(\AA^{2} \times 10^{3}\right)$ for $(R, S)-S 13 . U(e q)$ is defined as one third of the trace of the orthogonalized $U^{i j}$ tensor.

\begin{tabular}{|c|c|c|c|c|}
\hline & $x$ & $y$ & $z$ & $U(e q)$ \\
\hline$\overline{F(1)}$ & $2727(1)$ & $961(1)$ & $6152(1)$ & $60(1)$ \\
\hline$F(2)$ & 1463(1) & $684(1)$ & $7114(1)$ & $64(1)$ \\
\hline$F(3)$ & 3009(1) & $522(1)$ & 7637(1) & $67(1)$ \\
\hline$O(1)$ & $4187(1)$ & 2158(1) & $7136(1)$ & $53(1)$ \\
\hline $\mathrm{O}(2)$ & 1588(1) & $2639(1)$ & $6837(1)$ & $41(1)$ \\
\hline$N(1)$ & 3444(1) & $3648(1)$ & $7268(1)$ & $33(1)$ \\
\hline$C(1)$ & 2652(1) & $4325(1)$ & 7628(1) & $31(1)$ \\
\hline$C(2)$ & $3290(1)$ & 5194(1) & $8024(1)$ & $30(1)$ \\
\hline$C(3)$ & 4092(1) & $5262(1)$ & 7189(1) & $34(1)$ \\
\hline$C(4)$ & 4375(1) & 4193(1) & 6953(1) & $35(1)$ \\
\hline$C(5)$ & $4591(2)$ & 4026(2) & $5859(2)$ & $48(1)$ \\
\hline$C(6)$ & $5459(2)$ & 4659(2) & $5467(2)$ & 64(1) \\
\hline$C(7)$ & 3426(1) & 2660(1) & $7264(1)$ & $35(1)$ \\
\hline$C(8)$ & 2354(1) & 2173(1) & 7399(1) & $35(1)$ \\
\hline$C(9)$ & $2407(2)$ & 1076(1) & $7076(2)$ & $45(1)$ \\
\hline$C(10)$ & $1767(2)$ & $2809(2)$ & $5811(2)$ & 51(1) \\
\hline$C(11)$ & 2015(2) & 2202(1) & $8479(1)$ & 43(1) \\
\hline$C(12)$ & $1040(2)$ & $2506(2)$ & $8729(2)$ & $58(1)$ \\
\hline$C(13)$ & $743(3)$ & $2529(2)$ & $9714(2)$ & $83(1)$ \\
\hline$C(14)$ & $1410(4)$ & $2235(3)$ & $10433(2)$ & $93(1)$ \\
\hline$C(15)$ & $2373(3)$ & 1937(2) & 10189(2) & $88(1)$ \\
\hline$C(16)$ & 2691(2) & 1919(2) & $9214(2)$ & $65(1)$ \\
\hline$C(17)$ & 2648(1) & $6128(1)$ & $8119(1)$ & $33(1)$ \\
\hline$C(18)$ & 1596(2) & $6085(1)$ & $8296(2)$ & $43(1)$ \\
\hline$C(19)$ & $1015(2)$ & $6940(2)$ & $8351(2)$ & $56(1)$ \\
\hline$C(20)$ & $1470(2)$ & $7840(2)$ & $8236(2)$ & $63(1)$ \\
\hline$C(21)$ & $2510(2)$ & $7894(2)$ & $8084(2)$ & $62(1)$ \\
\hline$C(22)$ & $3102(2)$ & $7049(1)$ & $8031(2)$ & 47(1) \\
\hline$C(23)$ & $3777(1)$ & 4963(1) & $9025(1)$ & $32(1)$ \\
\hline$C(24)$ & 3248(2) & 4440(1) & 9740(1) & 42(1) \\
\hline$C(25)$ & $3673(2)$ & $4263(2)$ & 10658(1) & 51(1) \\
\hline$C(26)$ & $4631(2)$ & $4615(2)$ & 10884(2) & $54(1)$ \\
\hline$C(27)$ & 5167(2) & 5134(2) & 10189(2) & $56(1)$ \\
\hline$C(28)$ & $4751(2)$ & $5307(2)$ & 9266(1) & 44(1) \\
\hline
\end{tabular}


Table S3. Bond lengths $[\AA]$ and angles $\left[^{\circ}\right]$ for $(R, S)-S 13$.

\begin{tabular}{|c|c|c|c|}
\hline$F(1)-C(9)$ & $1.337(3)$ & $C(11)-C(16)$ & $1.391(3)$ \\
\hline$F(2)-C(9)$ & $1.344(2)$ & $C(12)-C(13)$ & $1.397(4)$ \\
\hline$F(3)-C(9)$ & $1.330(2)$ & $\mathrm{C}(12)-\mathrm{H}(12 \mathrm{~A})$ & 0.9300 \\
\hline $\mathrm{O}(1)-\mathrm{C}(7)$ & $1.218(2)$ & $C(13)-C(14)$ & $1.371(5)$ \\
\hline $\mathrm{O}(2)-\mathrm{C}(8)$ & $1.410(2)$ & $\mathrm{C}(13)-\mathrm{H}(13 \mathrm{~A})$ & 0.9300 \\
\hline $\mathrm{O}(2)-\mathrm{C}(10)$ & $1.437(3)$ & $C(14)-C(15)$ & $1.362(5)$ \\
\hline$N(1)-C(7)$ & $1.344(2)$ & $\mathrm{C}(14)-\mathrm{H}(14 \mathrm{~A})$ & 0.9300 \\
\hline$N(1)-C(1)$ & $1.470(2)$ & $C(15)-C(16)$ & $1.392(4)$ \\
\hline$N(1)-C(4)$ & $1.488(2)$ & $\mathrm{C}(15)-\mathrm{H}(15 \mathrm{~A})$ & 0.9300 \\
\hline$C(1)-C(2)$ & $1.544(2)$ & $\mathrm{C}(16)-\mathrm{H}(16 \mathrm{~A})$ & 0.9300 \\
\hline $\mathrm{C}(1)-\mathrm{H}(1 \mathrm{~A})$ & 0.9700 & $C(17)-C(22)$ & $1.392(3)$ \\
\hline $\mathrm{C}(1)-\mathrm{H}(1 \mathrm{~B})$ & 0.9700 & $C(17)-C(18)$ & $1.397(3)$ \\
\hline$C(2)-C(17)$ & $1.528(2)$ & $C(18)-C(19)$ & $1.391(3)$ \\
\hline$C(2)-C(23)$ & $1.537(2)$ & $\mathrm{C}(18)-\mathrm{H}(18 \mathrm{~A})$ & 0.9300 \\
\hline$C(2)-C(3)$ & $1.550(2)$ & $C(19)-C(20)$ & $1.371(4)$ \\
\hline$C(3)-C(4)$ & $1.535(2)$ & $\mathrm{C}(19)-\mathrm{H}(19 \mathrm{~A})$ & 0.9300 \\
\hline $\mathrm{C}(3)-\mathrm{H}(3 \mathrm{~A})$ & 0.9700 & $C(20)-C(21)$ & $1.375(4)$ \\
\hline $\mathrm{C}(3)-\mathrm{H}(3 \mathrm{~B})$ & 0.9700 & $\mathrm{C}(20)-\mathrm{H}(20 \mathrm{~A})$ & 0.9300 \\
\hline$C(4)-C(5)$ & $1.535(3)$ & $C(21)-C(22)$ & $1.387(3)$ \\
\hline $\mathrm{C}(4)-\mathrm{H}(4 \mathrm{~A})$ & 0.9800 & $\mathrm{C}(21)-\mathrm{H}(21 \mathrm{~A})$ & 0.9300 \\
\hline$C(5)-C(6)$ & $1.521(3)$ & $\mathrm{C}(22)-\mathrm{H}(22 \mathrm{~A})$ & 0.9300 \\
\hline $\mathrm{C}(5)-\mathrm{H}(5 \mathrm{~A})$ & 0.9700 & $C(23)-C(24)$ & $1.391(2)$ \\
\hline $\mathrm{C}(5)-\mathrm{H}(5 \mathrm{~B})$ & 0.9700 & $C(23)-C(28)$ & $1.395(3)$ \\
\hline $\mathrm{C}(6)-\mathrm{H}(6 \mathrm{~A})$ & 0.9600 & $C(24)-C(25)$ & $1.389(3)$ \\
\hline $\mathrm{C}(6)-\mathrm{H}(6 \mathrm{~B})$ & 0.9600 & $\mathrm{C}(24)-\mathrm{H}(24 \mathrm{~A})$ & 0.9300 \\
\hline $\mathrm{C}(6)-\mathrm{H}(6 \mathrm{C})$ & 0.9600 & $C(25)-C(26)$ & $1.375(3)$ \\
\hline$C(7)-C(8)$ & $1.560(2)$ & $\mathrm{C}(25)-\mathrm{H}(25 \mathrm{~A})$ & 0.9300 \\
\hline$C(8)-C(11)$ & $1.536(3)$ & $C(26)-C(27)$ & $1.374(3)$ \\
\hline$C(8)-C(9)$ & $1.558(2)$ & $\mathrm{C}(26)-\mathrm{H}(26 \mathrm{~A})$ & 0.9300 \\
\hline$C(10)-H(10 A)$ & 0.9600 & $C(27)-C(28)$ & $1.391(3)$ \\
\hline $\mathrm{C}(10)-\mathrm{H}(10 \mathrm{~B})$ & 0.9600 & $\mathrm{C}(27)-\mathrm{H}(27 \mathrm{~A})$ & 0.9300 \\
\hline$C(10)-H(10 C)$ & 0.9600 & $\mathrm{C}(28)-\mathrm{H}(28 \mathrm{~A})$ & 0.9300 \\
\hline$C(11)-C(12)$ & $1.381(3)$ & & \\
\hline $\mathrm{C}(8)-\mathrm{O}(2)-\mathrm{C}(10)$ & 119.01(15) & $C(4)-C(3)-C(2)$ & $105.09(12)$ \\
\hline $\mathrm{C}(7)-\mathrm{N}(1)-\mathrm{C}(1)$ & 128.01(15) & $\mathrm{C}(4)-\mathrm{C}(3)-\mathrm{H}(3 \mathrm{~A})$ & 110.7 \\
\hline $\mathrm{C}(7)-\mathrm{N}(1)-\mathrm{C}(4)$ & $120.81(14)$ & $\mathrm{C}(2)-\mathrm{C}(3)-\mathrm{H}(3 \mathrm{~A})$ & 110.7 \\
\hline $\mathrm{C}(1)-\mathrm{N}(1)-\mathrm{C}(4)$ & $111.02(13)$ & $\mathrm{C}(4)-\mathrm{C}(3)-\mathrm{H}(3 \mathrm{~B})$ & 110.7 \\
\hline$N(1)-C(1)-C(2)$ & $102.54(13)$ & $\mathrm{C}(2)-\mathrm{C}(3)-\mathrm{H}(3 \mathrm{~B})$ & 110.7 \\
\hline $\mathrm{N}(1)-\mathrm{C}(1)-\mathrm{H}(1 \mathrm{~A})$ & 111.3 & $\mathrm{H}(3 \mathrm{~A})-\mathrm{C}(3)-\mathrm{H}(3 \mathrm{~B})$ & 108.8 \\
\hline$C(2)-C(1)-H(1 A)$ & 111.3 & $N(1)-C(4)-C(5)$ & $110.87(14)$ \\
\hline $\mathrm{N}(1)-\mathrm{C}(1)-\mathrm{H}(1 \mathrm{~B})$ & 111.3 & $\mathrm{~N}(1)-\mathrm{C}(4)-\mathrm{C}(3)$ & $102.43(13)$ \\
\hline $\mathrm{C}(2)-\mathrm{C}(1)-\mathrm{H}(1 \mathrm{~B})$ & 111.3 & $C(5)-C(4)-C(3)$ & $112.86(15)$ \\
\hline $\mathrm{H}(1 \mathrm{~A})-\mathrm{C}(1)-\mathrm{H}(1 \mathrm{~B})$ & 109.2 & $\mathrm{~N}(1)-\mathrm{C}(4)-\mathrm{H}(4 \mathrm{~A})$ & 110.2 \\
\hline$C(17)-C(2)-C(23)$ & $108.77(13)$ & $\mathrm{C}(5)-\mathrm{C}(4)-\mathrm{H}(4 \mathrm{~A})$ & 110.2 \\
\hline$C(17)-C(2)-C(1)$ & $111.75(13)$ & $\mathrm{C}(3)-\mathrm{C}(4)-\mathrm{H}(4 \mathrm{~A})$ & 110.2 \\
\hline$C(23)-C(2)-C(1)$ & $112.18(13)$ & $C(6)-C(5)-C(4)$ & $113.18(17)$ \\
\hline$C(17)-C(2)-C(3)$ & $112.53(13)$ & $\mathrm{C}(6)-\mathrm{C}(5)-\mathrm{H}(5 \mathrm{~A})$ & 108.9 \\
\hline$C(23)-C(2)-C(3)$ & $112.59(13)$ & $\mathrm{C}(4)-\mathrm{C}(5)-\mathrm{H}(5 \mathrm{~A})$ & 108.9 \\
\hline$C(1)-C(2)-C(3)$ & $98.82(12)$ & $\mathrm{C}(6)-\mathrm{C}(5)-\mathrm{H}(5 \mathrm{~B})$ & 108.9 \\
\hline
\end{tabular}




\begin{tabular}{|c|c|c|c|}
\hline$C(4)-C(5)-H(5 B)$ & 108.9 & $C(18)-C(17)-C(2)$ & $121.35(15)$ \\
\hline $\mathrm{H}(5 \mathrm{~A})-\mathrm{C}(5)-\mathrm{H}(5 \mathrm{~B})$ & 107.8 & $\mathrm{C}(17)-\mathrm{C}(18)-\mathrm{H}(18 \mathrm{~A})$ & 119.6 \\
\hline$C(5)-C(6)-H(6 A)$ & 109.5 & $C(20)-C(19)-C(18)$ & $120.3(2)$ \\
\hline$C(5)-C(6)-H(6 B)$ & 109.5 & $C(20)-C(19)-H(19 A)$ & 119.9 \\
\hline $\mathrm{H}(6 \mathrm{~A})-\mathrm{C}(6)-\mathrm{H}(6 \mathrm{~B})$ & 109.5 & $C(18)-C(19)-H(19 A)$ & 119.9 \\
\hline$C(5)-C(6)-H(6 C)$ & 109.5 & $C(19)-C(20)-C(21)$ & $119.6(2)$ \\
\hline $\mathrm{H}(6 \mathrm{~A})-\mathrm{C}(6)-\mathrm{H}(6 \mathrm{C})$ & 109.5 & $\mathrm{C}(19)-\mathrm{C}(20)-\mathrm{H}(20 \mathrm{~A})$ & 120.2 \\
\hline $\mathrm{H}(6 \mathrm{~B})-\mathrm{C}(6)-\mathrm{H}(6 \mathrm{C})$ & 109.5 & $\mathrm{C}(21)-\mathrm{C}(20)-\mathrm{H}(20 \mathrm{~A})$ & 120.2 \\
\hline $\mathrm{O}(1)-\mathrm{C}(7)-\mathrm{N}(1)$ & $123.17(17)$ & $C(20)-C(21)-C(22)$ & $120.9(2)$ \\
\hline $\mathrm{O}(1)-\mathrm{C}(7)-\mathrm{C}(8)$ & $120.68(15)$ & $C(20)-C(21)-H(21 A)$ & 119.5 \\
\hline$N(1)-C(7)-C(8)$ & $116.12(15)$ & $C(22)-C(21)-H(21 A)$ & 119.5 \\
\hline $\mathrm{O}(2)-\mathrm{C}(8)-\mathrm{C}(11)$ & $107.68(14)$ & $C(21)-C(22)-C(17)$ & $120.3(2)$ \\
\hline $\mathrm{O}(2)-\mathrm{C}(8)-\mathrm{C}(9)$ & 107.97(14) & $\mathrm{C}(21)-\mathrm{C}(22)-\mathrm{H}(22 \mathrm{~A})$ & 119.8 \\
\hline$C(11)-C(8)-C(9)$ & 107.91(14) & $\mathrm{C}(17)-\mathrm{C}(22)-\mathrm{H}(22 \mathrm{~A})$ & 119.8 \\
\hline $\mathrm{O}(2)-\mathrm{C}(8)-\mathrm{C}(7)$ & $112.45(14)$ & $C(24)-C(23)-C(28)$ & $117.40(17)$ \\
\hline$C(11)-C(8)-C(7)$ & $111.17(14)$ & $C(24)-C(23)-C(2)$ & $121.37(15)$ \\
\hline$C(9)-C(8)-C(7)$ & 109.49(14) & $C(28)-C(23)-C(2)$ & $121.17(15)$ \\
\hline$F(3)-C(9)-F(1)$ & $106.82(17)$ & $C(25)-C(24)-C(23)$ & $121.42(19)$ \\
\hline$F(3)-C(9)-F(2)$ & 107.15(17) & $\mathrm{C}(25)-\mathrm{C}(24)-\mathrm{H}(24 \mathrm{~A})$ & 119.3 \\
\hline$F(1)-C(9)-F(2)$ & 106.02(16) & $\mathrm{C}(23)-\mathrm{C}(24)-\mathrm{H}(24 \mathrm{~A})$ & 119.3 \\
\hline$F(3)-C(9)-C(8)$ & $114.07(16)$ & $C(26)-C(25)-C(24)$ & $120.3(2)$ \\
\hline$F(1)-C(9)-C(8)$ & $113.13(15)$ & $\mathrm{C}(26)-\mathrm{C}(25)-\mathrm{H}(25 \mathrm{~A})$ & 119.8 \\
\hline$F(2)-C(9)-C(8)$ & 109.19(15) & $\mathrm{C}(24)-\mathrm{C}(25)-\mathrm{H}(25 \mathrm{~A})$ & 119.8 \\
\hline $\mathrm{O}(2)-\mathrm{C}(10)-\mathrm{H}(10 \mathrm{~A})$ & 109.5 & $C(27)-C(26)-C(25)$ & $119.26(19)$ \\
\hline $\mathrm{O}(2)-\mathrm{C}(10)-\mathrm{H}(10 \mathrm{~B})$ & 109.5 & $\mathrm{C}(27)-\mathrm{C}(26)-\mathrm{H}(26 \mathrm{~A})$ & 120.4 \\
\hline$H(10 A)-C(10)-H(10 B)$ & 109.5 & $\mathrm{C}(25)-\mathrm{C}(26)-\mathrm{H}(26 \mathrm{~A})$ & 120.4 \\
\hline $\mathrm{O}(2)-\mathrm{C}(10)-\mathrm{H}(10 \mathrm{C})$ & 109.5 & $C(26)-C(27)-C(28)$ & $120.8(2)$ \\
\hline $\mathrm{H}(10 \mathrm{~A})-\mathrm{C}(10)-\mathrm{H}(10 \mathrm{C})$ & 109.5 & $\mathrm{C}(26)-\mathrm{C}(27)-\mathrm{H}(27 \mathrm{~A})$ & 119.6 \\
\hline$H(10 B)-C(10)-H(10 C)$ & 109.5 & $\mathrm{C}(28)-\mathrm{C}(27)-\mathrm{H}(27 \mathrm{~A})$ & 119.6 \\
\hline$C(12)-C(11)-C(16)$ & $119.4(2)$ & $C(27)-C(28)-C(23)$ & $120.81(19)$ \\
\hline$C(12)-C(11)-C(8)$ & $120.67(18)$ & $\mathrm{C}(27)-\mathrm{C}(28)-\mathrm{H}(28 \mathrm{~A})$ & 119.6 \\
\hline$C(16)-C(11)-C(8)$ & 119.96(19) & $\mathrm{C}(23)-\mathrm{C}(28)-\mathrm{H}(28 \mathrm{~A})$ & 119.6 \\
\hline$C(11)-C(12)-C(13)$ & $120.0(3)$ & & \\
\hline $\mathrm{C}(11)-\mathrm{C}(12)-\mathrm{H}(12 \mathrm{~A})$ & 120.0 & & \\
\hline $\mathrm{C}(13)-\mathrm{C}(12)-\mathrm{H}(12 \mathrm{~A})$ & 120.0 & & \\
\hline$C(14)-C(13)-C(12)$ & $120.2(3)$ & & \\
\hline$C(14)-C(13)-H(13 A)$ & 119.9 & & \\
\hline$C(12)-C(13)-H(13 A)$ & 119.9 & & \\
\hline$C(15)-C(14)-C(13)$ & $120.0(3)$ & & \\
\hline$C(15)-C(14)-H(14 A)$ & 120.0 & & \\
\hline$C(13)-C(14)-H(14 A)$ & 120.0 & & \\
\hline$C(14)-C(15)-C(16)$ & $120.9(3)$ & & \\
\hline$C(14)-C(15)-H(15 A)$ & 119.5 & & \\
\hline$C(16)-C(15)-H(15 A)$ & 119.5 & & \\
\hline$C(15)-C(16)-C(11)$ & $119.5(3)$ & & \\
\hline$C(15)-C(16)-H(16 A)$ & 120.2 & & \\
\hline $\mathrm{C}(11)-\mathrm{C}(16)-\mathrm{H}(16 \mathrm{~A})$ & 120.2 & & \\
\hline$C(19)-C(18)-C(17)$ & $120.81(19)$ & & \\
\hline$C(19)-C(18)-H(18 A)$ & 119.6 & & \\
\hline$C(22)-C(17)-C(18)$ & $118.06(16)$ & & \\
\hline$C(22)-C(17)-C(2)$ & $120.59(16)$ & & \\
\hline
\end{tabular}


Symmetry transformations used to generate equivalent atoms:

Table S4. Anisotropic displacement parameters $\left(\AA^{2} \times 10^{3}\right)$ for $(R, S)-S 13$. The anisotropic displacement factor exponent takes the form: $-2 \pi^{2}\left[\eta^{2} a^{* 2} U^{11}+\ldots+2 h k a^{*} b^{*} U^{12}\right]$

\begin{tabular}{|c|c|c|c|c|c|c|}
\hline & $U^{11}$ & $U^{22}$ & U33 & $u^{23}$ & $U^{13}$ & $U^{12}$ \\
\hline$\overline{F(1)}$ & $69(1)$ & $51(1)$ & $62(1)$ & $-18(1)$ & $7(1)$ & $3(1)$ \\
\hline$F(2)$ & $60(1)$ & 47(1) & $84(1)$ & $-15(1)$ & $5(1)$ & $-19(1)$ \\
\hline$F(3)$ & $84(1)$ & $35(1)$ & $82(1)$ & $4(1)$ & $-18(1)$ & $7(1)$ \\
\hline$O(1)$ & $37(1)$ & $39(1)$ & $82(1)$ & $-5(1)$ & $2(1)$ & $8(1)$ \\
\hline $\mathrm{O}(2)$ & $33(1)$ & $44(1)$ & $45(1)$ & $0(1)$ & $-5(1)$ & $1(1)$ \\
\hline $\mathrm{N}(1)$ & $30(1)$ & $31(1)$ & $38(1)$ & $-3(1)$ & $3(1)$ & $0(1)$ \\
\hline$C(1)$ & $30(1)$ & $30(1)$ & $33(1)$ & $-3(1)$ & $0(1)$ & 1(1) \\
\hline$C(2)$ & $31(1)$ & $29(1)$ & $29(1)$ & $-2(1)$ & $3(1)$ & $0(1)$ \\
\hline$C(3)$ & $37(1)$ & $35(1)$ & $31(1)$ & $-3(1)$ & $5(1)$ & $-2(1)$ \\
\hline$C(4)$ & $31(1)$ & $36(1)$ & $36(1)$ & $-4(1)$ & $4(1)$ & $-1(1)$ \\
\hline$C(5)$ & $52(1)$ & $51(1)$ & $41(1)$ & $-13(1)$ & $13(1)$ & $-7(1)$ \\
\hline$C(6)$ & $69(2)$ & $70(1)$ & $52(1)$ & $-7(1)$ & $26(1)$ & $-11(1)$ \\
\hline$C(7)$ & 34(1) & $32(1)$ & $39(1)$ & $-3(1)$ & $-1(1)$ & $2(1)$ \\
\hline$C(8)$ & $35(1)$ & $30(1)$ & $40(1)$ & $-2(1)$ & $-2(1)$ & $1(1)$ \\
\hline $\mathrm{C}(9)$ & $48(1)$ & $35(1)$ & $54(1)$ & $-6(1)$ & $-1(1)$ & $-4(1)$ \\
\hline$C(10)$ & 59(1) & $52(1)$ & $43(1)$ & $0(1)$ & $-13(1)$ & $-2(1)$ \\
\hline$C(11)$ & $53(1)$ & $35(1)$ & $41(1)$ & $-2(1)$ & $3(1)$ & $-10(1)$ \\
\hline$C(12)$ & $58(1)$ & $58(1)$ & $56(1)$ & $-13(1)$ & 15(1) & $-17(1)$ \\
\hline$C(13)$ & $92(2)$ & $84(2)$ & $74(2)$ & $-28(2)$ & $38(2)$ & $-33(2)$ \\
\hline$C(14)$ & $144(3)$ & $88(2)$ & $48(1)$ & $-11(1)$ & 21(2) & $-52(2)$ \\
\hline$C(15)$ & $140(3)$ & $78(2)$ & $48(1)$ & $10(1)$ & $-15(2)$ & $-22(2)$ \\
\hline$C(16)$ & $90(2)$ & $55(1)$ & $48(1)$ & $7(1)^{\prime}$ & $-11(1)$ & $-2(1)$ \\
\hline$C(17)$ & $39(1)$ & $32(1)$ & $27(1)$ & $-3(1)$ & $0(1)^{\prime}$ & $3(1)$ \\
\hline$C(18)$ & 41(1) & $43(1)$ & $45(1)$ & $-4(1)$ & $5(1)$ & $6(1)$ \\
\hline$C(19)$ & $52(1)$ & $63(1)$ & $54(1)$ & $-7(1)$ & $2(1)$ & $21(1)$ \\
\hline$C(20)$ & $77(2)$ & $48(1)$ & $64(1)$ & $-9(1)$ & $-5(1)$ & 27(1) \\
\hline$C(21)$ & $81(2)$ & $34(1)$ & $72(2)$ & $-5(1)$ & $-4(1)$ & $7(1)$ \\
\hline$C(22)$ & $50(1)$ & $35(1)$ & $55(1)$ & $-2(1)$ & 1(1) & $0(1)$ \\
\hline$C(23)$ & $34(1)$ & $32(1)$ & $30(1)$ & $-2(1)$ & $0(1)$ & 4(1) \\
\hline$C(24)$ & 46(1) & $46(1)$ & $33(1)$ & $0(1)$ & $3(1)$ & $-3(1)$ \\
\hline$C(25)$ & $68(1)$ & $53(1)$ & $32(1)$ & $3(1)$ & $4(1)$ & $5(1)$ \\
\hline$C(26)$ & $66(1)$ & $59(1)$ & $37(1)$ & $-3(1)$ & $-13(1)$ & $19(1)$ \\
\hline$C(27)$ & 43(1) & $72(1)$ & $51(1)$ & $-9(1)$ & $-13(1)$ & $4(1)^{\prime}$ \\
\hline$C(28)$ & $38(1)$ & $51(1)$ & $41(1)$ & $-2(1)$ & $-3(1)$ & $-4(1)$ \\
\hline
\end{tabular}


Table S5. Hydrogen coordinates $\left(\times 10^{4}\right)$ and isotropic displacement parameters $\left(\AA^{2} \times 10^{3}\right)$ for $(R, S)$-S13.

\begin{tabular}{|c|c|c|c|c|}
\hline & $x$ & $y$ & $z$ & $\mathrm{U}(\mathrm{eq})$ \\
\hline $\mathrm{H}(1 \mathrm{~A})$ & 2247 & 4025 & 8144 & 38 \\
\hline $\mathrm{H}(1 \mathrm{~B})$ & 2202 & 4533 & 7101 & 38 \\
\hline $\mathrm{H}(3 \mathrm{~A})$ & 3803 & 5584 & 6617 & 41 \\
\hline $\mathrm{H}(3 \mathrm{~B})$ & 4690 & 5626 & 7403 & 41 \\
\hline $\mathrm{H}(4 \mathrm{~A})$ & 4966 & 3988 & 7346 & 41 \\
\hline $\mathrm{H}(5 \mathrm{~A})$ & 4761 & 3339 & 5757 & 58 \\
\hline $\mathrm{H}(5 \mathrm{~B})$ & 3974 & 4166 & 5488 & 58 \\
\hline $\mathrm{H}(6 \mathrm{~A})$ & 5585 & 4497 & 4793 & 95 \\
\hline $\mathrm{H}(6 \mathrm{~B})$ & 6067 & 4541 & 5845 & 95 \\
\hline $\mathrm{H}(6 \mathrm{C})$ & 5273 & 5340 & 5517 & 95 \\
\hline $\mathrm{H}(10 \mathrm{~A})$ & 1183 & 3129 & 5529 & 77 \\
\hline $\mathrm{H}(10 \mathrm{~B})$ & 1879 & 2192 & 5486 & 77 \\
\hline$H(10 C)$ & 2360 & 3218 & 5733 & 77 \\
\hline $\mathrm{H}(12 \mathrm{~A})$ & 582 & 2695 & 8242 & 69 \\
\hline$H(13 A)$ & 90 & 2745 & 9882 & 100 \\
\hline$H(14 A)$ & 1206 & 2239 & 11086 & 112 \\
\hline$H(15 A)$ & 2823 & 1743 & 10680 & 106 \\
\hline $\mathrm{H}(16 \mathrm{~A})$ & 3352 & 1720 & 9056 & 78 \\
\hline$H(18 A)$ & 1280 & 5479 & 8378 & 52 \\
\hline$H(19 A)$ & 314 & 6901 & 8467 & 67 \\
\hline $\mathrm{H}(20 \mathrm{~A})$ & 1079 & 8410 & 8260 & 76 \\
\hline$H(21 A)$ & 2820 & 8505 & 8017 & 75 \\
\hline $\mathrm{H}(22 \mathrm{~A})$ & 3805 & 7099 & 7935 & 56 \\
\hline$H(24 A)$ & 2596 & 4204 & 9601 & 50 \\
\hline $\mathrm{H}(25 \mathrm{~A})$ & 3307 & 3904 & 11121 & 61 \\
\hline$H(26 A)$ & 4912 & 4502 & 11501 & 65 \\
\hline $\mathrm{H}(27 \mathrm{~A})$ & 5817 & 5371 & 10338 & 67 \\
\hline $\mathrm{H}(28 \mathrm{~A})$ & 5127 & 5657 & 8803 & 52 \\
\hline
\end{tabular}


Table S6. Torsion angles [ $\left.{ }^{\circ}\right]$ for $(R, S)$-S13.

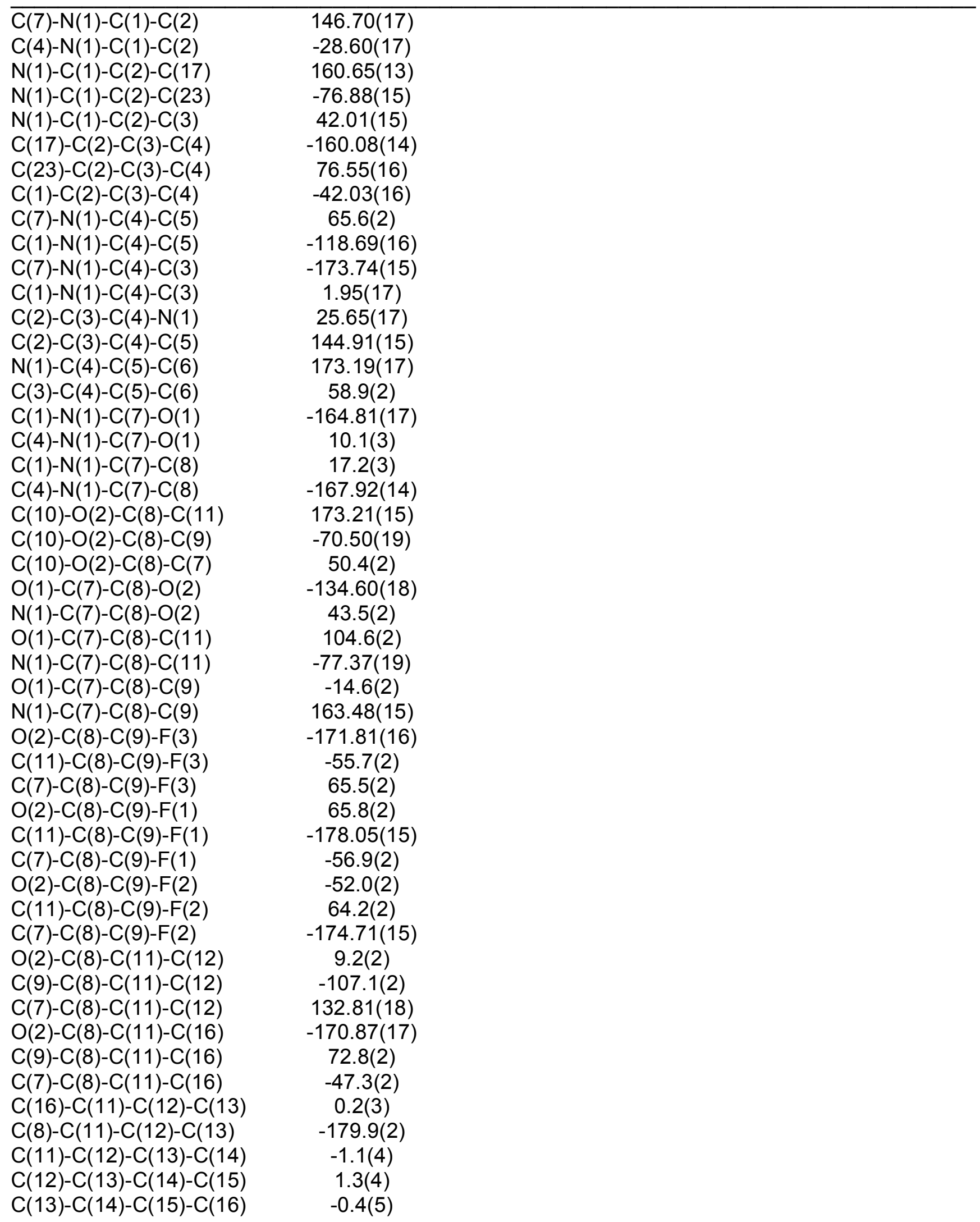




\begin{tabular}{|c|c|}
\hline$C(14)-C(15)-C(16)-C(11)$ & $-0.5(4)$ \\
\hline$C(12)-C(11)-C(16)-C(15)$ & $0.7(3)$ \\
\hline$C(8)-C(11)-C(16)-C(15)$ & $-179.3(2)$ \\
\hline$C(23)-C(2)-C(17)-C(22)$ & 84.42(19) \\
\hline$C(1)-C(2)-C(17)-C(22)$ & $-151.18(17)$ \\
\hline$C(3)-C(2)-C(17)-C(22)$ & $-41.0(2)$ \\
\hline$C(23)-C(2)-C(17)-C(18)$ & $-95.53(19)$ \\
\hline$C(1)-C(2)-C(17)-C(18)$ & $28.9(2)$ \\
\hline$C(3)-C(2)-C(17)-C(18)$ & $139.00(17)$ \\
\hline$C(22)-C(17)-C(18)-C(19)$ & $2.0(3)$ \\
\hline$C(2)-C(17)-C(18)-C(19)$ & $-178.05(17)$ \\
\hline$C(17)-C(18)-C(19)-C(20)$ & $-0.3(3)$ \\
\hline$C(18)-C(19)-C(20)-C(21)$ & $-1.2(4)$ \\
\hline$C(19)-C(20)-C(21)-C(22)$ & $1.0(4)$ \\
\hline$C(20)-C(21)-C(22)-C(17)$ & $0.7(4)$ \\
\hline$C(18)-C(17)-C(22)-C(21)$ & $-2.2(3)$ \\
\hline$C(2)-C(17)-C(22)-C(21)$ & $177.84(19)$ \\
\hline$C(17)-C(2)-C(23)-C(24)$ & $84.78(18)$ \\
\hline$C(1)-C(2)-C(23)-C(24)$ & $-39.4(2)$ \\
\hline$C(3)-C(2)-C(23)-C(24)$ & $-149.79(15)$ \\
\hline$C(17)-C(2)-C(23)-C(28)$ & $-92.46(18)$ \\
\hline$C(1)-C(2)-C(23)-C(28)$ & $143.40(16)$ \\
\hline$C(3)-C(2)-C(23)-C(28)$ & $33.0(2)$ \\
\hline$C(28)-C(23)-C(24)-C(25)$ & $-0.3(3)$ \\
\hline$C(2)-C(23)-C(24)-C(25)$ & $-177.59(16)$ \\
\hline$C(23)-C(24)-C(25)-C(26)$ & $0.8(3)$ \\
\hline$C(24)-C(25)-C(26)-C(27)$ & $-0.7(3)$ \\
\hline$C(25)-C(26)-C(27)-C(28)$ & $0.2(3)$ \\
\hline$C(26)-C(27)-C(28)-C(23)$ & $0.3(3)$ \\
\hline$C(24)-C(23)-C(28)-C(27)$ & $-0.3(3)$ \\
\hline$C(2)-C(23)-C(28)-C(27)$ & $177.05(18)$ \\
\hline
\end{tabular}

Symmetry transformations used to generate equivalent atoms:

\section{References}

1) Hickman, J. R.; Kenyon, J. J. Chem. Soc. 1995, 2051.

2) Zhang, Z.; Bender, C. F.; Widenhoefer, R. A. Org. Lett. 2007, 9, 2887.

3) Zhang, Z.; Widenhoefer, R. A. Angew. Chem. Int. Ed. 2007, 46, 283.

4) Nieto-Oberhuber, C.; López, S; Echavarren, A. M. J. Am. Chem. Soc. 2005, 127, 6178.

5) Zhang, Z.; Liu, C.; Kinder, R. E.; Han, X.; Qian, H.; Widenhoefer, R. A. J. Am. Chem. Soc. 2006, 128, 9066.

6) Cowie, J. S.; Landor, P. D.; Landor, S. R. J. Chem. Soc. Perkin Trans 1 1973, 7, 720.

7) Faulkner, D. J.; Petersen, M. R. J. Am. Chem. Soc. 1971, 93, 3766. 
8) (a) Olsson, L.-I.; Claesson, A. Acta Chem. Scand., Ser B 1977, 31, 614. (b) Claesson, A.; Olsson, L.-I. J. Am. Chem. Soc. 1979, 101, 7302. (c) Smith, R. A.; White, R. L.; Krantz, A. J. Med. Chem. 1988, 31, 1558.

9) Al-Dulayymi, J. R.; Baird, M. S. Tetrahedron 1990, 46, 5703.

10) Liu, C.; Han, X.; Wang, X.; Widenhoefer, R. A. J. Am. Chem. Soc. 2004, 126, 3700. 